\title{
NOTES ON SCREW THREADS.
}

\author{
By Mr. H. H. JEFFCOTT, OF THE \\ National Physical Laboratory, Tednington.
}

[Selected for Publication.]

\section{INDEX OF CONTENTS.}

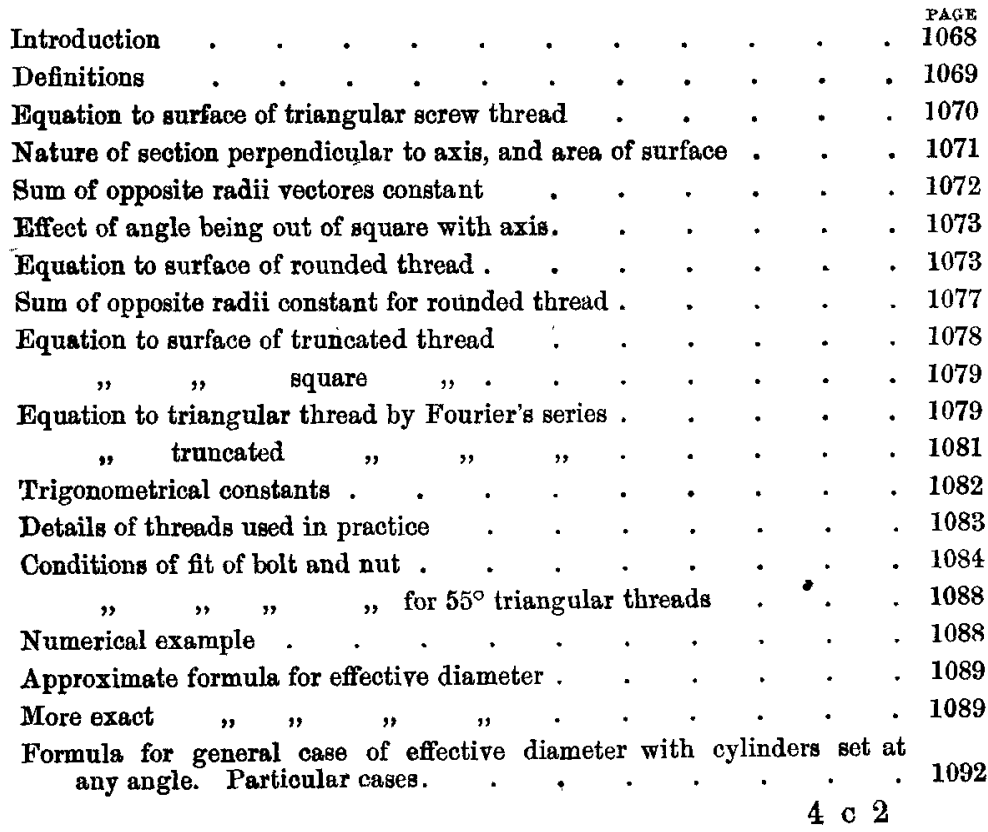


Limiting sizes of cylinders for measuring effective diameter of paqu rounded threads. $. \quad . \quad . \quad . \quad . \quad . \quad . \quad . \quad .1095$

Ditto, truncated threads . . . . . . . . . . . . 1096

Limiting sizes of cylinders for Whitworth threads. . . . . 1097

Formula for effective diameter of Whitworth threads . . . . . 1097

Effect of errors in various elements on effective diameter as measured . 1098

Effective diameter of Whitworth thread with cylinders perpendicular to axis of screw . . . . . . . . . . . 1100

Nature of shadow of a serew in parallel light . . . . . . . . 1100

Numerical example . . . . . . . . . . . . . 1102

Effect of tilt of cutting-tool on the thread derived. . . . . 1103

Table 1.-British Standard Whitworth Threads with additions . . 1107

"2. " " Fin $\Theta \quad " \quad$ ". . . 1108

Introduction.-The following notes contain an account of some theoretical aspects of screw threads, regarded partly from the standpoint of accuracy in the interpretation of results of measurement. and partly from that of correlation of errors in bolts and nuts.

Treated from a purely geometrical point of view the ordinary screw thread, on account of the complexity of its surface, presents insidious difficulties, so that it appeared advisable to resort to analysis and obtain the equation to its surfuce. This has been done in what follows in two different ways : one by a consideration of the method of generation of the screw thread itself, and the other by the use of Fourier's series.

A purely triangular (or V) thread is easiest to deal with, but the equations have been adapted as far as possible to the forms met with in practice, i.e., mainly with rounded or truncated crests and roote. The conditions of fit between a bolt and nut with triangular threads are examined, and although the results are not directly applicable to, say, a Whitworth thread, yet they can in any given case be readily modified to suit the different form of thread.

The measurement of the effective diameter of a screw is usually carried out by means of three small cylinders of equal diameters which are placed two on one side and one on the other side of the 
sorew. The distance over these cylinders is measured, and from it the effective diameter is deduced. Approximate formula have been given for this purpose based on the assumption that the section through the axis of the screw, in which measurement is made, cuts the cylinders in circles which touch the slopes of the thread. This is not quite true, and for accurate measurement is insufficient. The cylinders, being free, set themselves nearly along the rake of the thread, and this has to be taken into account.

Accordingly a more exact formula is given, and a similar case in which the cylinders are fixed perpendicular to the axial plane of measurement of the screw is also considered. The effect of small errors in the various elements of a screw on the interpretation of the results of measurement of effective diameter is exhibited as a formula. Various details as to sizes of cylinders suitable for any given case are added, and, further, many of the formula are expressed in forms ready for use with Whitworth threads.

The bounding curve of the shadow of a screw in parallel light is examined, and the limitations on methods of measurement on this basis are indicated. The effect of tilt of the cutting-tool on the shape of the thread derived is also briefly considered.

Tables of British Standard Whitworth, and Fine, Screw Threads are appended (pages 1107 and 1108), in which certain figures relative to effective diameter are for convenience worked out.

Definitions.-A screw thread is a surface determined by the following elements :--pitch, angle, full diameter, core diameter, and nature of crest and root.

These will be denoted by :-pitch $=p ;$ angle $=2 \alpha$; full diameter for triangular thread $=2 R$; core diameter for triangular thread $=2 R^{\prime}$; radius at crest and root (if rounded) $=\rho$.

The effective diameter is the length intercepted by the surface on a line cutting the axis at right angles, and is (in a perfect screw) equal to $R+R^{\prime}$.

It is proposed to obtain the equation to a screw thread surface, and for simplicity a purely triangular thread will first be dealt with ; i.e., $\rho=o$. 
Equation to surface of triangular screw thread.-Refer the surface to axes of cylindrical co-ordinates $z, r, \theta$, the axis of $z$ coinciding with the axis of the screw. (See Fig. 1.)

Fig. 1.
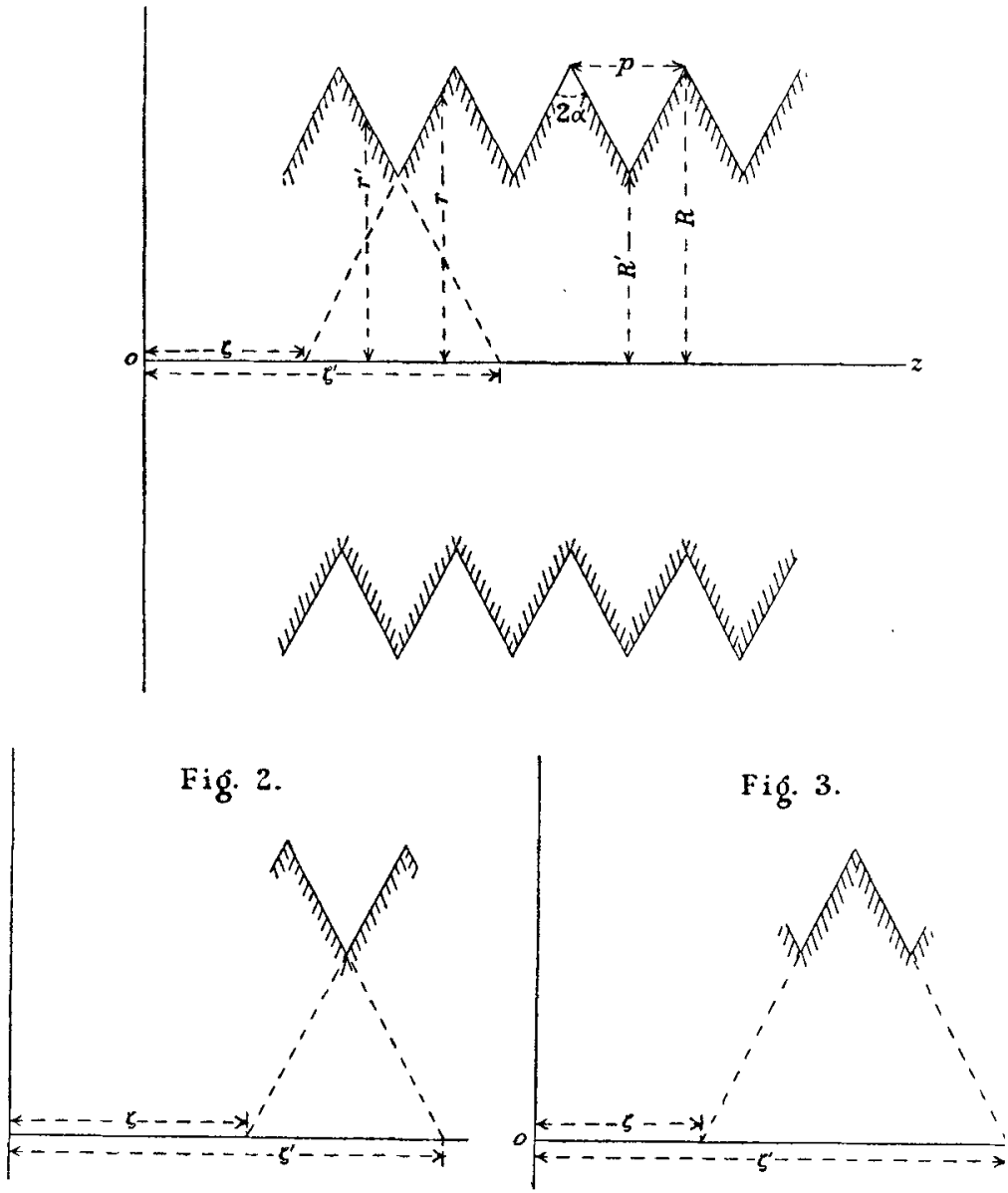

Regard the screw as a surface swept out by two lines intersecting the axis, which move along the axis as they rotate round it. 
Reckon $\theta$ positive in the direction of the thread which increases $z$.

Then $z-\zeta=r \tan a$ for one face of thread.

$\zeta^{\prime}-z^{\prime}=r^{\prime} \tan \alpha$ for other face of thread.

Now $\zeta^{\prime}=\zeta+2 R^{\prime} \tan \alpha$; and $\zeta=\frac{p \theta}{2 \pi}, \theta$ being the angle corresponding to $r$, and being zero when $\zeta=o$ (i.e., at origin).

Hence the co-ordinates of any point on the screw surface are connected by the following relations:-

$$
\left.\right|_{r=R} ^{r=R^{\prime}} z-\frac{p \theta}{2 \pi}=r \tan \alpha ;\left.\quad\right|_{r=R^{\prime}} ^{r=R} z-\frac{p \theta}{2 \pi}=\left(2 R^{\prime}-r\right) \tan \alpha .
$$

These two equations represent the surface.

They are obtained by regarding a $V$ groove swung around the axis, and moving forward at the same time along the axis. (See Fig. 2.)

Equation to surface of triangular screw thread.-Had the thread been regarded as formed by a $\Lambda$ projection swung around the axis, likewise moving forward, equivalent equations would have been obtained which may be written (see Fig. 3),

$$
\left.\right|_{r=R} ^{r=R^{\prime}} z-\frac{p \theta}{2 \pi}=r \tan \alpha ;\left.\quad\right|_{r=R^{\prime}} ^{r=R} z-\frac{p \theta}{2 \pi}=(2 R-r) \tan \alpha .
$$

Had the origin been chosen differently, the surface might also be represented by-

$$
\left.\right|_{r=R} ^{r=R^{\prime}} z-\frac{p \theta}{2 \pi}=\left(r-R^{\prime}\right) \tan \alpha ;\left.\right|_{r=R^{\prime}} ^{r=R} z-\frac{p \theta}{2 \pi}=\left(R^{\prime}-r\right) \tan \alpha .
$$

From geometry, $2 R=2 R^{\prime}+p \cot \alpha$.

Nature of section perpendicular to axis, and area of surface.-If a section be taken..perpendicular to the axis, i.e., take $z=$ constant, it is seen that each section has radii vectores which are obtained by 
subtracting those of an equiangular spiral from those of a circle, for angles from $o$ to $\pi$, and the reflection of this curve constitutes that remaining half of the section.

Thus for $z=k$ we have

$$
\begin{aligned}
r & =k \cot \alpha-\frac{p \cot \alpha}{2 \pi} \theta, \\
& =k^{\prime}-p^{\prime} \theta, \text { where } \zeta^{\prime} \text { and } p^{\prime} \text { are constants. }
\end{aligned}
$$

An expression for the area of cross-section perpendicular to the ¿xis of a screw with purely triangular threads may be readily deduced. It may be written

$$
\text { Area of cross section }=\frac{1}{4} \pi\left(R+R^{\prime}\right)^{2}+\frac{1}{48} \pi p^{2} \cot ^{2} \alpha .
$$

Or, the area of cross-section of a screw is greater than the area of a circle of diameter equal to the effective diameter of the screw by $\frac{1}{48} \pi p^{2} \cot ^{2} a$.

The area of the surface of a screw with purely triangular threads may also be obtained.

Taking an axial length of the screw of one pitch, the area of the surface is

$$
\begin{aligned}
\operatorname{Sec} \alpha & \left\{R \sqrt{ } 4 \pi^{2} R^{\overline{2}}+p^{2} \cos ^{2} a-R^{\prime} \sqrt{4 \pi^{2} R^{\prime 2}+p^{2} \cos ^{2} a}\right\} \\
& +\frac{p^{2} \cos \alpha}{2 \pi} \log _{e} \frac{2 \pi R+\sqrt{4 \pi^{2} R^{2}+p^{2} \cos ^{2} \alpha}}{2 \pi R^{\prime}+\sqrt{4 \pi^{2} R^{22}+p^{2} \cos ^{2} \alpha}}
\end{aligned}
$$

An approximate expression for the area of surface of a screw for an axial length of one pitch is

$$
\pi p\left(R+R^{\prime}\right) \operatorname{cosec} \alpha \text {. }
$$

Sum of opposite radii vectores constant.-If $r_{1}$ and $r_{2}$ be twc opposite radii vectores (i.e., have an angle $\pi$ between them) it is readily seen that $r_{1}+r_{2}=$ constant. This may be established from the equations, thus-

Let the section be $z=k$.

Then $k-\frac{p \theta_{1}}{2 \pi}=r_{1} \tan \alpha$.

$$
k-\frac{p\left(\theta_{1}+\pi\right)}{2 \pi}=\left(2 R^{\prime}-r_{2}\right) \tan \alpha .
$$




$$
\begin{aligned}
\therefore \quad\left(r_{1}+r_{2}\right) \tan \alpha & =2 R^{\prime} \tan \alpha+\frac{p}{2} . \\
\therefore \quad r_{1}+r_{2} & =2 R^{\prime}+\frac{1}{2} p \cot \alpha . \\
= & R+R^{\prime} . \\
= & \text { constant. }
\end{aligned}
$$

Effect of angle being out of square with axis.-If one imagines the angle out of square with the axis, let $a, \alpha^{\prime}$ be the angles the sides of the thread make with the perpendicular to the axis. Then the equations become (see Fig. 4, page 1074)-

$$
\begin{aligned}
& \mid \begin{array}{l}
r=R^{\prime} \\
r=R
\end{array}-\frac{p \theta}{2 \pi}=r \tan \alpha ; \\
& \left.\right|_{r=R^{\prime}} ^{r=R} \quad z-\frac{p \theta}{2 \pi}=\left(R^{\prime}-r\right) \tan \alpha^{\prime}+R^{\prime} \tan \alpha .
\end{aligned}
$$

Similarly, if the second formula had been chosen (page 1071), the right-hand side is obtained by writing $R$ for $R^{\prime}$ in the above.

Equation to surface of rounded thread.-A more general case will next be considered, in which $\rho$ is not zero.

Imagine a new thread derived from the purely triangular thread by rounding off the crest and root by circles, of radius $\rho$, which touch the sides of the thread. Let $m$ be the fraction of the depth of the thread that is thus cut off the top and also off the bottom of the thread. (See Fig. 5, page 1074.)

Then, for the straight line portion, the surface equation becomes

$$
\mid \begin{array}{c|c}
r=R^{\prime}+\rho \cos \alpha \cot \alpha \\
z-\frac{p \theta}{Z \pi}=r \tan \alpha ; \\
r=R-\rho \cos \alpha \cot \alpha
\end{array} \quad \begin{gathered}
r=R-\rho \cos \alpha \cot \alpha \\
\quad-\frac{p \theta}{2 \pi}=\left(2 R^{\prime}-r\right) \tan \alpha . \\
r=R^{\prime}+\rho \cos \alpha \cot \alpha
\end{gathered}
$$

The circular parts of the surface have now to be expressed in the formula.

From $r=R-\rho(\operatorname{cosec} \alpha-1)$ to $r=R-\rho \cos \alpha \cot \alpha$. 

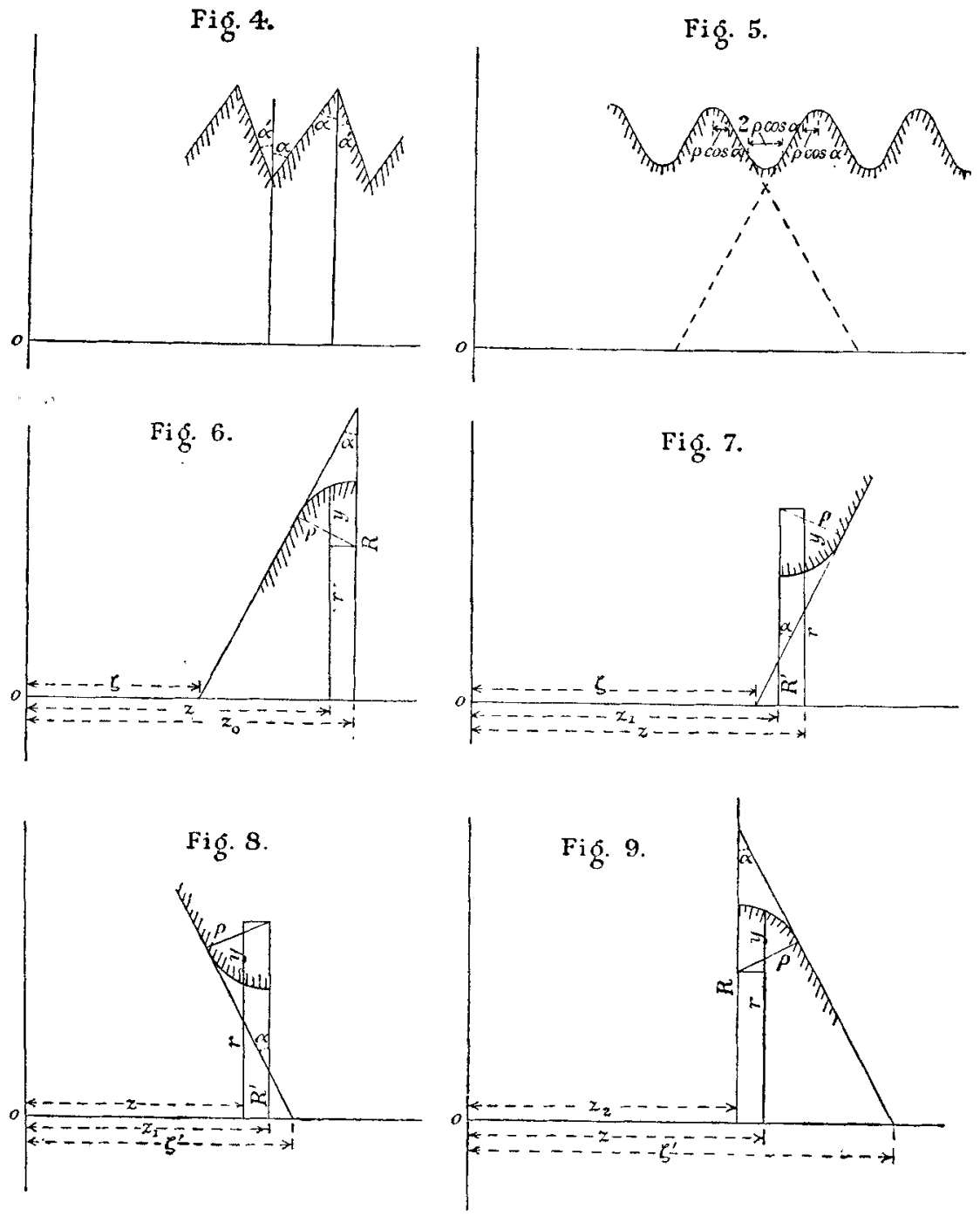
We have (see Fig. 6)

$$
\begin{aligned}
& \rho^{2}=y^{2}+\left(z_{0}-z\right)^{2} . \\
& y=r-R+\rho \operatorname{cosec} \alpha . \\
& z_{0}=R \tan \alpha+\zeta .
\end{aligned}
$$

$\therefore \zeta-z+R \tan a=\sqrt{2 \rho(R-\gamma) \operatorname{cosec} \alpha-(R-r)^{2}-p^{2} \cot ^{2} \alpha}$.

Now

$$
\zeta=\frac{p \theta}{2 \pi} ; \rho=\frac{m p \cos \alpha}{2(1-\sin \alpha)} .
$$

Hence from $r=R-\rho(\operatorname{cosec} \alpha-1)$ to $r=R-\rho \cos \alpha \cot$ the surface is represented by

$$
\mid \begin{aligned}
& r=R-\rho \cos \alpha \cot \alpha . \\
& \quad z-\frac{p \theta}{2 \pi}=R \tan \alpha-\sqrt{2 \rho(R-r) \operatorname{cosec} \alpha-(R-r)^{2}-\rho^{2}} \cot ^{2} \alpha . \\
& r=R-\rho(\operatorname{cosec} \alpha-1) .
\end{aligned}
$$

From $r=R^{\prime}+\rho \cos \alpha \cot \alpha$ to $r=R^{\prime}+\rho(\operatorname{cosec} \alpha-1)$. For portion of the root we have similarly (see Fig. 7)

$$
\begin{aligned}
& \rho^{2}=y^{2}+\left(z-z_{1}\right)^{2} . \\
& y=R^{\prime}+\rho \operatorname{cosec} \alpha-r . \\
& z_{1}=R^{\prime} \tan \alpha+\zeta . \\
\therefore & z-R^{\prime} \tan a-\zeta=\sqrt{2 \rho\left(r-R^{\prime}\right) \operatorname{cosec} \alpha-\left(r-R^{\prime}\right)^{2}-\rho^{2} \cot ^{2} \alpha .}
\end{aligned}
$$

Hence from $r=R^{\prime}+\rho \cos a \cot a$ to $r=R^{\prime}+\rho(\operatorname{cosec} a-1)$ the equation to the surface is

$$
\mid \begin{aligned}
& r=R^{\prime}+\rho(\operatorname{cosec} \alpha-1) . \\
& \quad z-\frac{p \theta}{2 \pi}=R^{\prime} \tan \alpha+\sqrt{2 \rho\left(r-R^{\prime}\right) \operatorname{cosec} \alpha-\left(r-R^{\prime}\right)^{2}-\rho^{2} \cot ^{2} \alpha .} \\
& r=R^{\prime}+\rho \cos \alpha \cot \alpha .
\end{aligned}
$$

From $r=R^{\prime}+\rho(\operatorname{cosec} \alpha-1)$ to $r=R^{\prime}+\rho \cos \alpha \cot \alpha$. For the aecond portion of the root, we have (see Fig. 8)

$$
\begin{aligned}
& \rho^{2}=y^{2}+\left(z_{1}-z\right)^{2} . \\
& y=R^{\prime}+\rho \operatorname{cosec} \alpha-r . \\
& z_{1}=\zeta^{\prime}-R^{\prime} \tan \alpha . \\
& \zeta^{\prime}=\zeta+2 R^{\prime} \tan \alpha . \\
\therefore \quad \zeta^{\prime} & =z-R^{\prime} \tan \alpha=\sqrt{2} \rho\left(r-R^{\prime}\right) \operatorname{cosec} \alpha-\left(r-R^{\prime}\right)^{2}-\rho^{2} \cot ^{2} \alpha .
\end{aligned}
$$

Hence from $r=R^{\prime}+\rho(\operatorname{cosec} a-1)$ to $r=R^{\prime}+\rho \cos \alpha \cot \alpha$ the equation to the surface is 


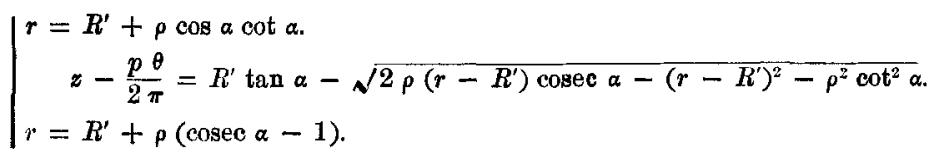

From $r=R-\rho \cos \alpha \cot \alpha$ to $r=R-\rho(\operatorname{cosec} \alpha-1)$.

For the second portion of the crest, we have (see Fig. 9, page 1074)

$$
\begin{aligned}
& \rho^{2}=y^{2}+\left(z-z_{2}\right)^{2} . \\
& y=r-R+\rho \operatorname{cosec} \alpha . \\
& z_{z}=\zeta^{\prime}-R \tan \alpha . \\
& \zeta^{\prime}=\zeta+2 R^{\prime} \tan \alpha .
\end{aligned}
$$

$\therefore z-\zeta-\left(2 R^{\prime}-R\right) \tan a=\sqrt{2} \rho(\dot{R-r}) \operatorname{cosec} \alpha-(R-r)^{2}-\rho^{2} \cot ^{2} \alpha$.

Hence from $r=R-\rho \cos \alpha \cot \alpha$ to $r=R-\rho(\operatorname{cosec} a-1)$ the equation to the surface is

$$
\mid \begin{aligned}
& r=R-\rho(\operatorname{cosec} \alpha-1) . \\
& \quad z-\frac{p \theta}{2 \pi}=\left(2 R^{\prime}-R\right) \tan \alpha+\sqrt{ }^{2} \rho(R-r) \operatorname{cosec} a-(R-r)^{2}-\rho^{2} \cot ^{2} a . \\
& r=R-\rho \cos \alpha \cot \alpha .
\end{aligned}
$$

Hence the complete equation to surface of a serew with triangular threads rounded off at root and crest to circles, of radius $\rho$, touching the slopes, is

$$
\begin{aligned}
& \mid \begin{aligned}
r= & R-\rho \cos \alpha \cot \alpha . \\
& z-\frac{p \theta}{2 \pi}=R \tan a-\sqrt{2} \rho(R-r) \operatorname{cosec} \alpha-(R-r)^{2}-\rho^{2} \cot ^{2} a . \\
r= & R-\rho(\operatorname{cosec} \alpha-1) .
\end{aligned} \\
& \mid \begin{aligned}
r= & R^{\prime}+\rho \cos \alpha \cot a . \\
& z-\frac{p \theta}{2 \pi}=r \tan \alpha . \\
r= & R-\rho \cos a \cot \alpha . \\
& z-\frac{p \theta}{2 \pi}=R^{\prime} \tan \alpha+\sqrt{2 \rho\left(r-R^{\prime}\right) \operatorname{cosec} \alpha-\left(r-h^{\prime}\right)^{2}-\rho^{2} \cot ^{2} \alpha} \\
r= & R^{\prime}+\rho \cos \alpha \cot \alpha . \\
r= & R^{\prime}+\rho \cos \alpha \cot \alpha . \\
& z-\frac{p \theta}{2 \pi}=R^{\prime} \tan \alpha-\sqrt{2 \rho\left(r-R^{\prime}\right) \operatorname{cosec} \alpha-\left(r-h^{\prime}\right)^{2}-\rho^{2} \cot ^{2} \alpha .} \\
r= & R^{\prime}+\rho(\operatorname{cosec} \alpha-1) .
\end{aligned}
\end{aligned}
$$




$$
\begin{aligned}
& \mid \begin{aligned}
r= & R-\rho \cos \alpha \cot \alpha . \\
& z-\frac{p \theta}{2 \pi}=\left(2 R^{\prime}-r\right) \tan \alpha . \\
r= & R^{\prime}+\rho \cos \alpha \cot \alpha .
\end{aligned} \\
& \mid \begin{aligned}
r= & R-\rho(\operatorname{cosec} \alpha-1) . \\
& z-\frac{p \theta}{2 \pi}=\left(2 R^{\prime}-R\right) \tan \alpha+\sqrt{2 \rho(R-r) \operatorname{cosec} \alpha-(R-r)^{2}-\rho^{2} \cot ^{2} \alpha .} \\
r= & R-\rho \cos \alpha \cot \alpha .
\end{aligned}
\end{aligned}
$$

If the root and crest be each rounded off to circles, touching the slopes of the thread, cutting away a fraction $m$ of the depth of triangular thread in each case, then

$$
\rho=2 \frac{m}{2(1-\sin \alpha)} .
$$

Sum of opposite radii constant for rounded thread.-If $r_{1}$ and $r_{2}$ be two opposite radii vectores in any section (i.e., have an angle $\pi$ between them), it can be shown that $r_{1}+r_{2}$ is constant for the rounded thread. For the right line portion of the thread this has already been shown (page 1072).

For one curved part we have, putting $z=k$,

$$
\begin{gathered}
k-\frac{p \theta_{1}}{2 \pi}=R^{\prime} \tan \alpha+\sqrt{2 \rho\left(r_{1}-\overline{R^{\prime}}\right) \operatorname{cosec} \alpha-\left(r_{1}-R^{\prime}\right)^{2}-\rho^{2} \cot ^{2} \alpha .} \\
k-\frac{p\left(\theta_{1}+\pi\right)}{2 \pi}=\left(2 R^{\prime}-h^{2}\right) \tan a \\
+\sqrt{2 \rho\left(K-r_{2}\right) \operatorname{cosec} \alpha-\left(K-r_{2}\right)^{2}-\rho^{2} \cot ^{2} \alpha .}
\end{gathered}
$$

Subtracting, and remembering that $R-R^{\prime}=\frac{1}{2} p \cot a$, we find

$$
\begin{aligned}
& \quad \sqrt{2 \rho\left(r_{1}-h^{\prime}\right) \operatorname{cosec} \alpha-\left(r_{1}-R^{\prime}\right)^{2}-\rho^{2} \cot ^{2} \alpha} \\
& =\sqrt{2 \rho\left(R-r_{2}\right) \operatorname{cosec} a-\left(R-r_{2}\right)^{2}-\rho^{2} \cot ^{2} a .} \\
& \text { Hence } \quad \quad 2 \rho \operatorname{cosec} \alpha\left\{\left(R+R^{\prime}\right)-\left(r_{1}+r_{2}\right)\right\} \\
& =\left\{R-R^{\prime}+r_{1}-r_{2}\right\}\left\{\left(R+R^{\prime}\right)-\left(r_{1}+r_{2}\right)\right\} . \\
& \text { Thug } r_{1}+r_{2}=R+R^{\prime} \text {, or } 2 \rho \operatorname{cosec} a=R-R^{\prime}+r_{1}-r_{2} .
\end{aligned}
$$

The latter solution is not in general true, so we have

$$
r_{1}+r_{2}=R+R^{\prime}=\text { constant. }
$$

Similarly the same result for the remaining opposite curved parts may be established. 
Equation to surface of truncated thread.-The case will next be considered in which the triangular thread is truncated by right lines parallel to the axis, at both root and crest. (See Fig. 10.)

Let $m$ be the fraction of depth of thread that is thus cut off the root and also off the crest.

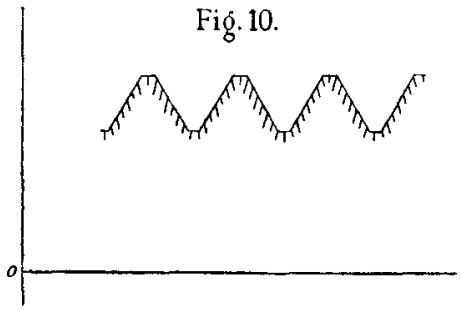

Fió. 12.
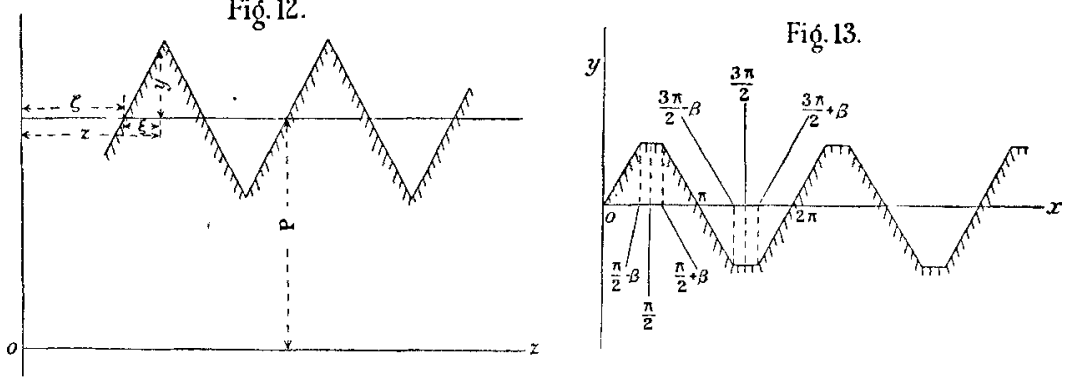

The equations to the slopes of the thread are the same as before. The equations to the truncated parts are

and

$$
r=\mathrm{constant}=R-\frac{1}{2} m p \cot a .
$$

and

$$
r=\text { constant }=R^{\prime}+\frac{1}{2} m p \cot \alpha .
$$

$$
r=\text { constant }=R-\frac{1}{2} m p \cot \alpha .
$$

Hence the complete surface is represented by

$$
\mid \begin{gathered}
r=R-\frac{1}{2} m p \cot \alpha . \\
r=R-\frac{1}{2} m p \cot \alpha . \\
r=R-\frac{1}{2} m p \cot \alpha .
\end{gathered}
$$




$$
\begin{aligned}
& \mid \begin{array}{c}
r=R^{\prime}+\frac{1}{2} m p \cot \alpha . \\
z-\frac{p \theta}{2 \pi}=r \tan \alpha . \\
r=R-\frac{1}{2} m p \cot \alpha .
\end{array} \\
& \mid \begin{array}{c}
r=R^{\prime}+\frac{1}{2} m p \cot \alpha . \\
r=R^{\prime}+\frac{1}{2} m p \cot \alpha . \\
r=R^{\prime}+\frac{1}{2} m p \cot \alpha .
\end{array} \\
& \mid \begin{array}{c}
r=R-\frac{1}{2} m p \cot \alpha . \\
z-\frac{p \theta}{2 \pi}=\left(2 R^{\prime}-r\right) \tan \alpha . \\
r=R^{\prime}+\frac{1}{2} m p \cot \alpha . \\
r=R-\frac{1}{2} m p \cot \alpha . \\
r=R-\frac{1}{2} m p \cot \alpha . \\
r=R-\frac{1}{2} m p \cot \alpha .
\end{array}
\end{aligned}
$$

Equation to surface of square thread.-For a square thread similarly it is found that the surface is represented by

$$
\left|\begin{array}{l|l}
r=r_{1} \\
r=r_{1}
\end{array} r=r_{1} ; \quad\right| \begin{aligned}
& r=r_{2} \\
& r=r_{2}
\end{aligned} r=r_{2},
$$

$2 r_{1}$ and $2 r_{2}$ being the full and core diameters.

Also from $r=r_{1}$ to $r=r_{2}$, we have

$$
\mid \begin{aligned}
& r=r_{2} \\
& r=r_{1}
\end{aligned} \quad \frac{p \theta}{2 \pi},
$$

and from $r=r_{2}$ to $r=r_{1}$, we have

$$
\left\{\begin{array}{l}
r=r_{1} \\
r=r_{2}
\end{array} z=\frac{p \theta}{2 \pi} .\right.
$$

Equation to triangular thread by Fourier's series.-An equation might have been obtained to the surface of a screw thread otherwise by means of Fourier's series.

Consider a purely triangular thread. (See Fig. 11, page 1078.) 
Let $y=f(x)$ represent the recurring series of right lines. We have now to express

$$
f(x)=a_{1} \sin x+a_{2} \sin 2 x+a_{3} \sin 3 x+\ldots \ldots
$$

Here in general for the qth term

$$
a_{q}=\frac{2}{\pi} \int_{0}^{\pi} f(x) \sin q x d x .
$$

Now $y=k x$ from $o$ to $\frac{\pi}{2}$; and $y=k(\pi-x)$ from $\frac{\pi}{2}$ to $\pi$.

$$
\begin{gathered}
\therefore a_{q}=\frac{2}{\pi} \int_{0}^{\frac{\pi}{2}} k x \sin q x d x+\frac{2}{\pi} \int_{\frac{\pi}{2}}^{\pi} k(\pi-x) \sin q x d x \\
=\frac{4 k}{\pi q^{2}} \sin q \frac{\pi}{2} .
\end{gathered}
$$

Hence $y=\frac{4 k}{\pi}\left[\frac{1}{1^{2}} \sin x-\frac{1}{3^{2}} \sin 3 x+\frac{1}{5^{2}} \sin 5 x-\ldots ..\right]$.

Let $\xi=$ length along axis of $x$. Then $\frac{\xi}{p}=\frac{x}{2 \pi}$, if $p$ is the pitch.

$$
\therefore \quad x^{\prime}=\frac{2 \pi \xi}{p} \text {. }
$$

Further, $y=\xi \cot a$, and $y=k x$.

$$
\therefore k=\frac{p \cot a}{2 \pi} \text {. }
$$

\section{Hence}

$y=\frac{2 p \cot \alpha}{\pi^{2}}\left[\frac{1}{1^{2}} \sin \frac{2 \pi \xi}{p}-\frac{1}{3^{2}} \sin \frac{3 \times 2 \pi \xi}{p}+\frac{1}{5^{2}} \sin \frac{5 \times 2 \pi \xi}{p}-\ldots\right]$.

$\checkmark=\frac{p \theta}{2 \pi}$, the origin being suitably chosen. (See Fig. 12, page 1078.)

$$
z-\zeta=\xi, \quad y=r-\frac{R+R^{\prime}}{2}=r-P, \text { if } 2 P=R+R^{\prime} .
$$

\section{Hence}

$$
\begin{aligned}
\frac{\pi^{2}(r-P)}{2 p \cot a}= & \frac{1}{1^{2}} \sin \left(\frac{2 \pi z}{p}-\theta\right)-\frac{1}{3^{2}} \sin 3\left(\frac{2 \pi z}{p}-\theta\right) \\
& +\frac{1}{5^{2}} \sin 5\left(\frac{2 \pi z}{p}-\theta\right)-\ldots \ldots
\end{aligned}
$$


This is an equation to the surface in $z, r, \theta$ co-ordinates, and the series is in general rapidly convergent. For many purposes the first four terms will suffice, so that the surface of a triangular screw thread may be expressed by

$$
\begin{gathered}
\frac{\pi^{2}(r-P)}{2 p \cot a}=\sin \left(\frac{2 \pi z}{p}-\theta\right)-\frac{1}{9} \sin 3\left(\frac{2 \pi z}{p}-\theta\right) \\
+\frac{1}{25} \sin 5\left(\frac{2 \pi z}{p}-\theta\right)-\frac{1}{49} \sin 7\left(\frac{2 \pi z}{p}-\theta\right) .
\end{gathered}
$$

Equation to truncated thread by Fourier's series.-Dimilarly an equation to the surface of a truncated triangular thread may be derived by Fourier's series.

Let $y=f(x)$ represent the series of right lines, including truncations, as in Fig. 13 (page 1078).

Express

$$
f(x)=a_{1} \sin x+a_{2} \sin 2 x+a_{3} \sin 3 x+\ldots
$$

Then

$$
a_{q}=\frac{2}{\pi} \int_{0}^{\pi} f(\dot{x}) \sin _{r} q x d x
$$

Here,

$$
\begin{aligned}
& \text { from } o \text { to } \frac{\pi}{2}-\beta, \quad y=k x \\
& \text { from } \frac{\pi}{2}-\beta \text { to } \frac{\pi}{2}+\beta, \quad y=0 \\
& \text { from } \frac{\pi}{2}+\beta \text { to } \pi, \quad y=k(\pi-x)
\end{aligned}
$$

Also

$$
\begin{gathered}
k=\frac{p \cot \alpha}{2 \pi}, \text { and } e=k\left(\begin{array}{l}
\pi \\
2
\end{array}-\beta\right) . \\
\therefore \quad \frac{\pi}{2} a_{i g}=\int_{0}^{\frac{\pi}{2}-\beta} k x \sin q x d x+\int_{\frac{\pi}{2}-\beta}^{\frac{\pi}{2}+\beta} c \sin q x d x \\
+\int_{\frac{\pi}{2}+\beta}^{\pi} k(\pi-x) \sin q x d x .
\end{gathered}
$$


Now

$$
\begin{gathered}
\int \sin q x d x=-\frac{1}{q} \cos q x . \\
\int x \sin q x d x=-\frac{1}{q} x \cos q x+\frac{1}{q^{2}} \sin q x . \\
\therefore a_{q}=\frac{4 k}{\pi q^{2}} \times \sin q \frac{\pi}{2} \times \cos q \beta .
\end{gathered}
$$

If $m$ be the fraction of depth of thread cut off the top and also off the bottom of the trianglar thread, then $\beta=\pi m$.

Also $y=r-P$; so we find, as on page 1080 .

$$
\begin{gathered}
\frac{\pi^{2}(r-P)}{2 p \cot \alpha}-\frac{1}{1^{2}} \cos m \pi \cdot \sin \left(\frac{2 \pi z}{p}-\theta\right)-\frac{1}{3^{2}} \cos 3 m \pi \cdot \sin 3\left(\frac{2 \pi z}{p}-\theta\right) \\
+\frac{1}{5^{2}} \cos 5 m \pi \cdot \sin 5\left(\frac{2 \pi z}{p}-\theta\right)-\ldots \ldots \\
+\frac{1}{q^{2}} \cos q m \pi \times \sin q \frac{\pi}{2} \times \sin q\left(\frac{2 \pi z}{p}-\theta\right)+\ldots \ldots
\end{gathered}
$$

For many purposes the first four terms will be sufficient, when

\begin{tabular}{|c|c|c|c|}
\hline Angle $2 \alpha=$ & $55^{\circ}$ & $60^{\circ}$ & $47 \frac{1}{2}^{\circ}$ \\
\hline $\sin a=$ & 0.4617486 & 0.5000000 & 0.4027467 \\
\hline $\cos \alpha=$ & 0.8870108 & $0 \cdot 8660254$ & 0.9153115 \\
\hline $\tan a=$ & 0.5205671 & 0.5773503 & 0.4400105 \\
\hline $\operatorname{cosec} \alpha=$ & $2 \cdot 1656806$ & $2 \cdot 0000000$ & $2 \cdot 4829503$ \\
\hline $\sec \alpha=$ & $1 \cdot 1273819$ & $1 \cdot 1547005$ & $1 \cdot 0925243$ \\
\hline $\cot \alpha=$ & $1 \cdot 9209821$ & $1 \cdot 7320508$ & $2 \cdot 2726729$ \\
\hline
\end{tabular}
the following may be taken

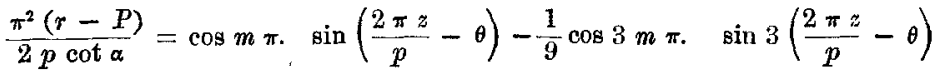

$$
\begin{aligned}
& +\frac{1}{25} \cos 5 m \pi . \quad \sin 5\left(\frac{2 \pi z}{p}-\theta\right)-\frac{1}{49} \cos 7 m \pi . \quad \sin 7\left(\frac{2 \pi z}{p}-\theta\right) .
\end{aligned}
$$

Trigonometrical Constants.-The following Table of Constants is useful :- 
Detrils of threads used in practice.-The following Table gives the forms of the principal screw threads in use, and indicates how they are derived from the purely triangular thread.

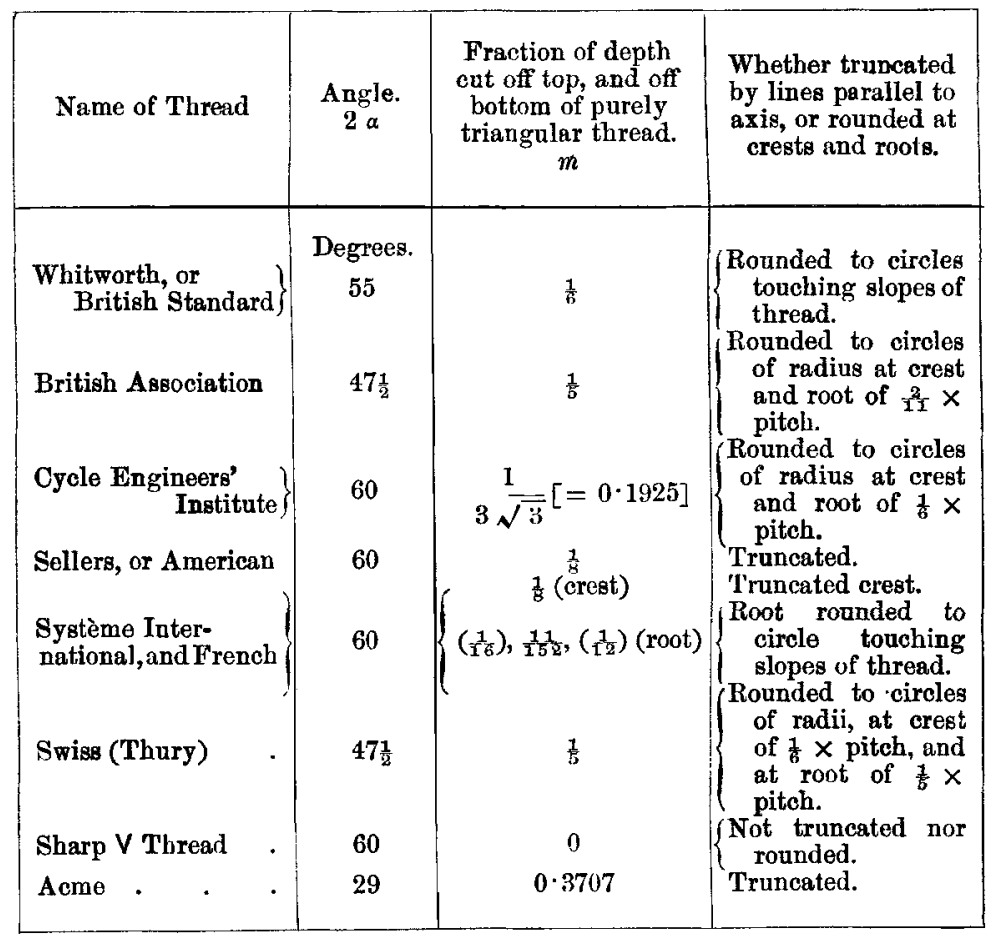


Conditions of fit of bolt and nut.-Some of the preceding results will now be applied to the determination of the conditions of fit of a bolt and nut. Purely triangular threads will be considered, and differences in pitch, angle, diameters, and out of squareness of angle with axis will be taken into account.

For the nut let $\alpha, a^{\prime}$ be the angles between the sides of thread and the perpendicular to the axis; $2 R$ and $2 R^{\prime}$ the full and core diameters (of the triangular threads); $p$ the pitch; and $w$ the number of threads of the nut engaged.

For the bolt let $a_{1}, \alpha_{1}^{\prime}, R_{1}, R_{1}^{\prime} p_{1}, w_{1}$ represent similar quantities.

Then the surface equations may be written :-

$$
\begin{gathered}
z-\frac{p \theta}{2 \pi}=r \tan \alpha ; z-\frac{p \theta}{2 \pi}=R \tan a+(R-r) \tan \alpha^{\prime} . \\
z_{1}-\frac{p_{1} \theta_{1}}{2 \pi}=r_{1} \tan \alpha_{1}+\zeta ; z_{1}-\frac{p_{1} \theta_{1}}{2 \pi}=R_{1} \tan \alpha_{1}+\left(R_{1}-r_{1}\right) \tan \alpha_{1}^{\prime}+\zeta .
\end{gathered}
$$

where $\zeta$ accounts for the difference in positions of origin of $z$ axis, if $\theta$ be the same for both bolt and nut.

Put then $\theta=\theta_{1}$, and we get

$$
\begin{aligned}
z-r \tan a & =\frac{z_{1}-\zeta-r_{1} \tan \alpha_{1}}{p_{1}} ; \\
p & = \\
z-R \tan a-(R-r) \tan \alpha^{\prime} & ={ }_{p}-\zeta-R_{1} \tan a_{1}-\left(R_{1}-r_{1}\right) \tan \alpha_{1}^{\prime} .
\end{aligned}
$$

Let bolt and nut be in contact at $z=o$ on first face, and at $z=v p$ on second face.

Hence

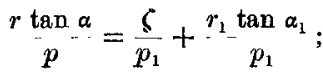

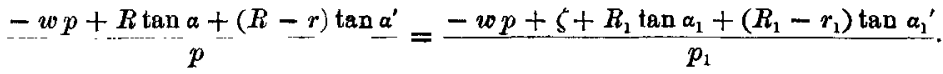

Then too $r=r_{1}$, so that

$$
\begin{gathered}
r\left(\frac{\tan \alpha}{p} \cdot{ }^{\alpha}-\frac{\tan \alpha_{1}}{p_{1}}\right)=\frac{\zeta}{p_{1}} ; \\
\frac{R\left(\tan \alpha+\tan \alpha^{\prime}\right)}{p}-\frac{R_{1}\left(\tan \alpha_{1}+\tan a_{1}^{\prime}\right)}{p_{1}}-r\left(\begin{array}{c}
\tan \alpha^{\prime} \\
p
\end{array}-\frac{\tan \alpha_{1}{ }^{\prime}}{p_{1}}\right) \\
=\frac{\zeta}{p_{1}}+\frac{w\left(p_{1}-p\right)}{p_{1}} .
\end{gathered}
$$


In these two equations the values of $r$ may be different; call them $r$ and $r$. Fliminating $\zeta$ we obtain

$$
\begin{gathered}
\frac{R-r}{p} \tan a+\frac{R-r^{\prime}}{p} \tan \alpha^{\prime}-\frac{R_{1}-r}{p_{1}} \tan a_{1} \\
-{ }_{1} \frac{r^{\prime}}{p_{1}} \tan a_{1}^{\prime}={ }^{w\left(p_{1}-p\right)} \\
p_{1} \\
=\frac{w_{1}\left(p_{1}-p\right)}{p} .
\end{gathered}
$$

This equation governs the fit.

Four different cases may be separated:-

$$
\begin{array}{ll}
\text { (1) } a>a_{1}, & a^{\prime}>a_{1}{ }^{\prime} \\
\text { (2) } a>a_{1}, & a^{\prime}<a_{1}{ }^{\prime} \\
\text { (3) } a<\alpha_{1}, & a^{\prime}>a_{1}{ }^{\prime} \\
\text { (4) } a<a_{1}, & a^{\prime}<a_{1}{ }^{\prime}
\end{array}
$$

The corresponding geometrical conditions of fit may be depicted thuis (see Fig. 14) :-

Fig. 14.
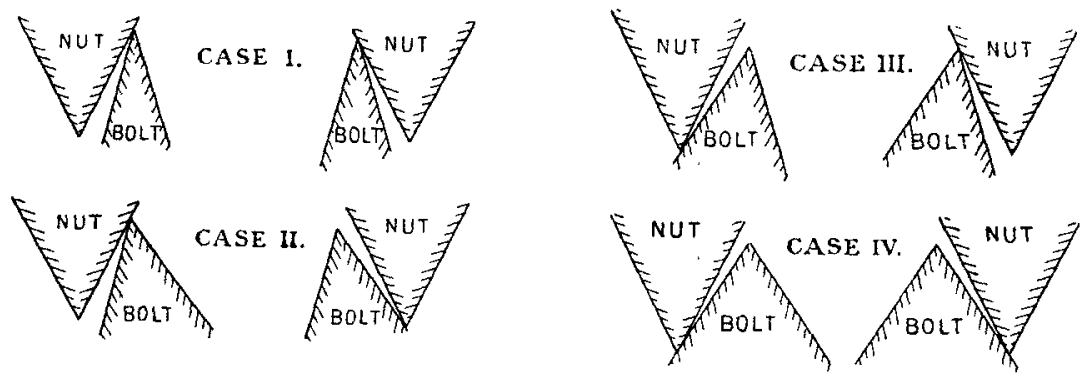

The values of $r$ and $r$ in the four cases are
(1) $r=R_{1}, \quad r^{\prime}=R_{1}$
(2) $r=R_{1}, \quad r^{\prime}=R^{\prime}$
(3) $r=R^{\prime}, \quad r^{\prime}=l_{1}$
(4) $r=k^{\prime}, \quad r^{\prime}=R^{\prime}$ 
Hence we find

$$
\frac{R-R_{1}}{p}\left(\tan a+\tan a^{\prime}\right)=\frac{w\left(p_{1}-p\right)}{p_{1}}
$$

$$
\underset{p}{R-R_{1}} \tan \alpha+\frac{R-R^{\prime}}{p} \tan \alpha^{\prime}-\frac{R_{1}-R^{\prime}}{p_{1}} \tan \alpha_{1}^{\prime}=\frac{w\left(p_{1}-p\right)}{p_{1}}
$$

(3) $\quad \frac{R-R^{\prime}}{p} \tan \alpha+\frac{R-R_{1}}{p} \tan \alpha^{\prime}-\frac{R_{1}-R^{\prime}}{p_{1}} \tan \alpha_{1}=\frac{w\left(p_{1}-p\right)}{p_{1}}$

(4) $\quad \frac{R-R^{\prime}}{p}\left(\tan \alpha+\tan \alpha^{\prime}\right)-\frac{R_{1}-R^{\prime}}{p_{1}}\left(\tan \alpha_{1}+\tan \alpha_{1}{ }^{\prime}\right)={ }^{w\left(p_{1}-p\right)} p_{1}$

If one proceeds to approximations, the errors being supposed all small, and put $2 \gamma=$ correct angle; $\tan \gamma=t ; a=\gamma+$; $a^{\prime}=\gamma+\boldsymbol{\bullet} ; \alpha_{1}=\gamma+\epsilon_{1} ; \alpha_{1}{ }^{\prime}=\gamma+\epsilon_{1}{ }^{\prime} ; R-R_{1}=\rho ; R^{\prime}-R_{1}{ }^{\prime}=\rho^{\prime}$; $\rho+\rho^{\prime}=\Delta ; R-R^{\prime}=h ; p_{1}-p=\sigma ; x=\frac{\sigma}{p}$.

Then $\tan (\gamma+\epsilon)=\frac{\tan \gamma+\tan \epsilon}{1-\tan \gamma \cdot \tan \epsilon}={ }_{1+\epsilon}^{t+\epsilon}=(t+\epsilon)(1+\epsilon t)=t+\epsilon+\epsilon t^{2}$ approximately.

Hence one obtains, omitting products of small quantities

$$
2 \rho t=w \sigma
$$

$$
2 \rho t+h\left\{t x-\left(1+t^{2}\right)\left(\epsilon_{1}^{\prime}-\epsilon^{\prime}\right)\right\}=w \sigma
$$

$$
2 \rho t+h\left\{t x-\left(1+t^{2}\right)\left(\epsilon_{1}-\epsilon\right)\right\}=w \sigma
$$

Now $h=\frac{p}{2} t ; x=\frac{\sigma}{p}$. Hence

$$
\begin{aligned}
& 2 \rho t=w \sigma \\
& 2 \rho t=\left(w-\frac{1}{2}\right) \sigma+\frac{1}{2} p\left(t+\frac{1}{t}\right)\left(\epsilon_{1}^{\prime}-\epsilon^{\prime}\right) \\
& 2 \rho t=\left(w-\frac{1}{2}\right) \sigma+\frac{1}{2} p\left(t+\frac{1}{t}\right)\left(\epsilon_{1}-\epsilon\right) \\
& 2 \rho t=(w-1) \sigma+\frac{1}{2} p\left(t+\frac{1}{t}\right)\left(\epsilon_{1}+\epsilon_{1}^{\prime}-\epsilon-\epsilon^{\prime}\right)
\end{aligned}
$$

Or, finally

$$
\begin{aligned}
& 2 \rho t=w \sigma \\
& 2 \rho t=\left(w-\frac{1}{2}\right) \sigma+\frac{1}{2} p\left(t+\frac{1}{t}\right)\left(\alpha_{1}{ }^{\prime}-\alpha^{\prime}\right) \\
& 2 \rho t=\left(w-\frac{1}{2}\right) \sigma+\frac{1}{2} p\left(t+\frac{1}{t}\right)\left(\alpha_{1}-\alpha\right) \\
& 2 \rho t=(w-1) \sigma+\frac{1}{2} p\left(t+\frac{1}{t}\right)\left(a_{1}+a_{1}{ }^{\prime}-a-\alpha^{\prime}\right)
\end{aligned}
$$


These four equations govern the fit of bolt and nut, and take account of differences of pitch, full dismeter, angle, and squareness of thread.

Similar equations may be written giving the difference in core diameters instead of in full diameters. These can be readily derived from the preceding by aid of the obvious relation.

$$
2 \rho t=2 \rho^{\prime} t-\sigma-\frac{1}{2} p\left(t+\frac{1}{t}\right)\left(\alpha+\alpha^{\prime}-a_{1}-a_{1}^{\prime}\right) .
$$

Making this substitution one finds

$$
\begin{aligned}
& 2 \rho^{\prime} t=(w+1) \sigma+\frac{1}{2} p\left(t+\frac{1}{t}\right)\left(\alpha+a^{\prime}-a_{1}-a_{1}{ }^{\prime}\right) \\
& 2 \rho^{\prime} t=\left(w+\frac{1}{2}\right) \sigma+\frac{1}{2} p\left(t+\frac{1}{t}\right)\left(\alpha-a_{1}\right) \\
& 2 \rho^{\prime} t=\left(w+\frac{1}{2}\right) \sigma+\frac{1}{2} p\left(t+\frac{1}{t}\right)\left(a^{\prime}-a_{1}{ }^{\prime}\right) \\
& 2 \rho^{\prime} t=w \sigma
\end{aligned}
$$

If, again, it is desired to introduce the difference in effective diameters $\Delta$ instead of the difference in full diameters, we have, since $\Delta=\rho+\rho^{\prime}$,

$$
\begin{aligned}
& \Delta \cdot t=\left(w+\frac{1}{2}\right) \sigma+\frac{1}{4} p\left(t+\frac{1}{t}\right)\left(a+a^{\prime}-a_{1}-a_{1}^{\prime}\right) \\
& \Delta \cdot t=w \sigma+\frac{1}{4} p\left(t+\frac{1}{t}\right)\left(\alpha-a_{1}+a_{1}{ }^{\prime}-a^{\prime}\right) \\
& \Delta \cdot t=w \sigma+\frac{1}{4} p\left(t+\frac{1}{t}\right)\left(a_{1}-a+\alpha^{\prime}-a_{1}^{\prime}\right) \\
& \Delta \cdot t=\left(w-\frac{1}{2}\right) \sigma+\frac{1}{4} p\left(t+\frac{1}{t}\right)\left(a_{1}+\alpha_{1}^{\prime}-\alpha-\alpha^{\prime}\right)
\end{aligned}
$$

In the preceding, $t=$ tangent of correct half angle.

$$
\begin{aligned}
& \frac{1}{2}\left(a-a^{\prime}\right)=\text { angle of out of square of nut thread with axis } \\
& \frac{1}{2}\left(a_{1}-a_{1}{ }^{\prime}\right)=\quad, \quad, \quad \text { " bolt " " } \\
& 2 \rho=\text { difference in full diameters of bolt and nut } \\
& \mathbf{2} \rho^{\prime}=\quad, \quad, \text { core } \quad ", \quad, \\
& \Delta=\text { " ", effective " , " , } \\
& \sigma=", \quad \text { pitch of bolt and nut }
\end{aligned}
$$

$w=$ number of threads of nut engaged. $p=$ pitch of nut. 
Conditions of fit of bolt and nut.-If the out of squareness of thread with axis of screw is omitted, the number of cases is reduced is two, viz., (1) and (4) of the above.

Let the difference in angle between bolt and nut be numerically represented by $\beta$.

Then we have

Case I. Nut angle greater than bolt angle.

$$
\begin{aligned}
& 2 \rho t=w \sigma \\
& 2 \rho^{\prime} t=(w+1) \sigma+\frac{1}{2} p \beta\left(t+\frac{1}{t}\right) \\
& \Delta t=\left(w+\frac{1}{2}\right) \sigma+\frac{1}{4} p \beta\left(t+\frac{1}{t}\right)
\end{aligned}
$$

Case II. Nut angle less than bolt angle.

$$
\begin{aligned}
& 2 \rho t=(w-1) \sigma+\frac{1}{2} p \beta\left(t+\frac{1}{t}\right) \\
& 2 \rho^{\prime} t=w \sigma \\
& \Delta t=\left(w-\frac{1}{2}\right) \sigma+\frac{1}{2} p \beta\left(t+\frac{1}{t}\right)
\end{aligned}
$$

Conditions of fit of bolt and nut for $55^{\circ}$ triangular threads.-The above results may be adapted for a $55^{\circ}$ triangular thread by writing $t=0 \cdot 52$.

Further, let $\beta^{\circ}$ be the difference in angle expressed in degrees. The following are then obtained

Case I. Nut angle greater than bolt angle.

$$
\begin{aligned}
& 2 \rho=1.92 w \sigma \\
& 2 \rho^{\prime}=1.92(w+1) \sigma+0.0409 p \beta^{\circ} \\
& \Delta=1.92\left(w+\frac{1}{2}\right) \sigma+0.0205 p \beta^{\circ}
\end{aligned}
$$

Case II. Nut angle less than bolt angle.

$$
\begin{aligned}
& 2 \rho=1.92(w-1) \sigma+0.0409 p \beta^{\circ} \\
& 2 \rho^{\prime}=1.92 w \sigma \\
& \Delta=1.92\left(w-\frac{1}{2}\right) \sigma+0.0205 p \beta^{\circ}
\end{aligned}
$$

Numerical example.-As a numerical example a bolt and nut with $55^{\circ}$ triangular thread, and 8 threads per inch may be taken.

Let there be a difference in piteh between them of $0.0004^{\prime \prime}$ (i.e., 0.0032 " pitch difference per inch), and let the difference in angle be $3^{\circ}$. 
Let it be required that $v=8$; then the allowances required on the diameters may be estimated as follows:-

Case I. Nut angle greater than bolt angle by $3^{\circ}$.

Allowance on full diameter $=2 \rho=1.92 \times 8 \times 0.0004=0.0061^{\prime \prime}$

$$
\begin{aligned}
& \text { " core } " \quad=2 \rho^{\prime}=1.92 \times 9 \times 0.0004+0.0409 \times 0.125 \times 3 \\
& =0.0069+0.0153=0.0222^{\prime \prime}
\end{aligned}
$$

Allowance on effective diameter $=\Delta=1.92 \times 8 \frac{1}{2} \times 0.0004+0.0205 \times 0.125$

$$
\times 3=0.0065+0.0076=0.0141^{\prime \prime}
$$

Case II. Nut angle less than bolt angle by $3^{\circ}$.

Allowance on full diameter $=2 \rho=0.0054+0.0153=0.0207^{\prime \prime}$

$$
\begin{aligned}
& \Rightarrow \quad \text { core } " \quad=2 \rho^{\prime}=0.0061 \\
& " \quad \text { effective, } \quad=\Delta=c .0058+0.0076=0.0134 "
\end{aligned}
$$

Approximate formula for effective diameter.-It is proposed now to pass on to the consideration of some points relative to the measurement of effective diameter of screws.

The measurement of effective diameter of a screw is usually made by using three circular cylinders of equal diameters which are arranged one on one side and two on the other side of the screw. The distance over the cylinders is measured, and also the diameter of the cylinders, and from these the effective diameter may be determined in terms of the pitch and angle. (See Fig. 15, page 1090.)

Let $T$ be the distance over the oylinders, and $c$ be the radius of the cylinders.

Then

$$
\frac{1}{2} T=R^{\prime}+e(1+\operatorname{cosec} \alpha) .
$$

If $2 P$ is the effective diameter,

$$
\begin{gathered}
P=\frac{1}{2}\left(R+R^{\prime}\right)=R^{\prime}+\frac{1}{4} p \cot \alpha . \\
\therefore \quad 2 P=T+\frac{1}{2} p \cot \alpha-2 c(1+\operatorname{cosec} \alpha) .
\end{gathered}
$$

More exact formula for effective diameter.-This value is, however, not quite correct, as the cylinders set themselves practically along the rake of the thread, and their sections in the axial plane are ellipses. The condition of things are shown in the figure. (See Fig. 16, page 1090.) 
Fig. 15.

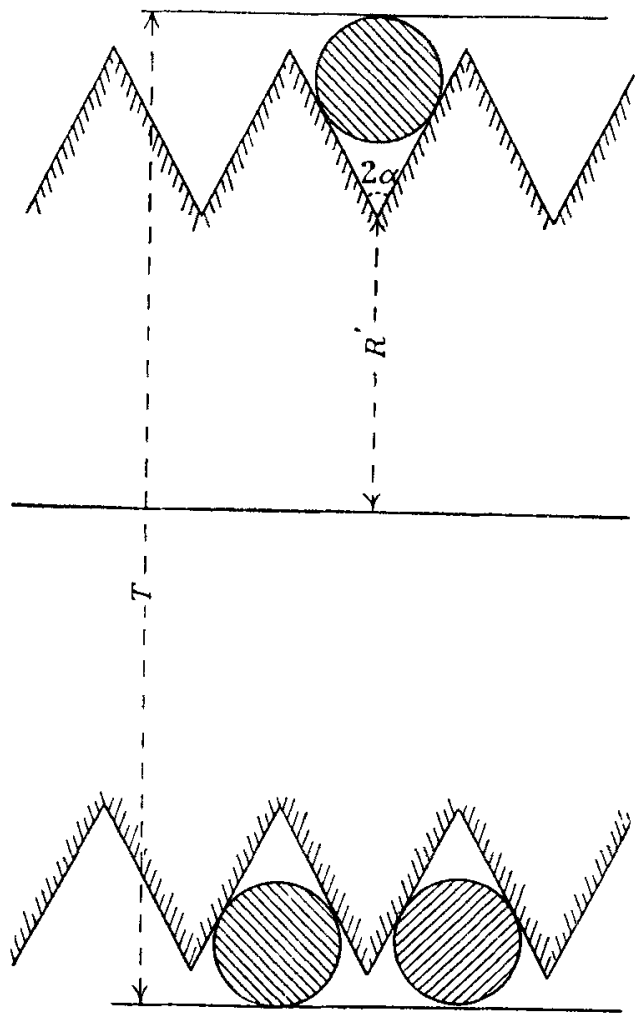

Fig. 16.

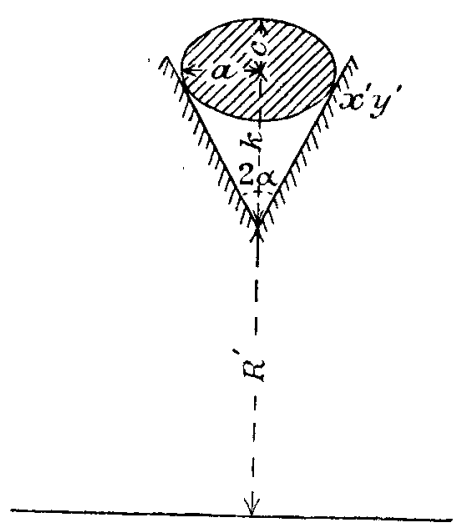

Fig 17.

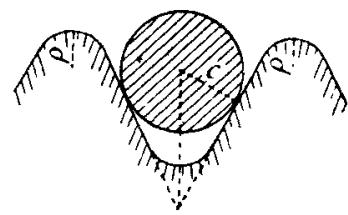


Here

$$
\frac{1}{2} T=R^{\prime}+c+k \text {. }
$$

Let the point of contact of ellipse with the thread be $x^{\prime} y$ referred to the axes of the ellipse, $c$ being now the semi-axis minor.

Let $a$ be the semi-axis major.

Then

$$
\frac{x^{\prime 2}}{a^{2}}+\frac{y^{\prime 2}}{c^{2}}=1
$$

Also

Hence

$$
k y^{\prime}=c^{2} ; \text { and } \frac{x^{\prime}}{k-y^{\prime}}=\tan \alpha .
$$

$$
k^{2}=a^{2} \cot ^{2} \alpha+c^{2} .
$$

The equation to surface of screw being $z-\frac{p \theta}{2 \pi}=r \tan a$ it is seen that, for a certain radius vector $r$, the tangent of the angle of rake $=$ $\frac{d z}{r d \theta}=\frac{p}{2 \pi r}=\mu$ say.

Also

$$
\begin{aligned}
a & =c \times \text { secant of angle of rake. } \\
\therefore \quad a & =c \sqrt{1+\mu^{2}} .
\end{aligned}
$$

Hence, eliminating $a$ and $k$ we obtain

$$
\frac{1}{2} T=R^{\prime}+c\left\{1+\sqrt{\operatorname{cosec}^{2} a+\mu^{2} \cot ^{2} \alpha}\right\} \text {. }
$$

Now

$$
P=R^{\prime}+\frac{1}{p} p \cot \alpha .
$$

$$
\therefore \quad 2 P=T+\frac{1}{2} p \cot \alpha-2 c\left\{1+\sqrt{\operatorname{cosec}^{2} \alpha+\mu^{2}} \cot ^{2} a\right\} \text {. }
$$

This formula gives the value of the effective diameter, acconnt baving been taken of the skewing of the cylinders along the rake of the thread.

Since $\mu^{2}$ is usually very small, the formula may be written thus$2 P=T+\frac{1}{2} p \cot \alpha-2 o\left\{1+\operatorname{cosec} \alpha+\frac{1}{2} \mu^{2} \cos \alpha \cot \alpha\right\}$.

This gives a value for effective diameter smaller than that in the approximate formula by $c \mu^{2} \cos \alpha \cot \alpha$.

The value of this difference for all sizes of British Standard Whitworth Threads is nearly 0.0002 ". 
Formula for general case of effective diameter with cylinders set at any angle. Particular cases.-More generally, consider the case in which the cylinders are set at any angle $\beta$ with the normal to the axial plane section of the screw.

To solve this case, one may write the equations of the screw and cylinder, and express the condition that they touch, i.e., have a common tangent plane at a common point.

Screw is $U=z-\frac{p \theta}{2 \pi}-r \tan \alpha$.

Cylinder is

$\boldsymbol{V}=z-k-r \sin \theta \tan \beta-\sec \beta \sqrt{c^{2}-\left(r_{\prime \prime}-r \cos \theta\right)^{2}} ;$

where $k=R^{\prime} \tan a ; \beta$ is the angle the axis of the cylinder makes with the normal to the axial plane section of the screw, $r_{o}$ is radius vector to centre of section of cylinder in axial plane of screw.

Now the tangent plane at $x^{\prime} y^{\prime} z^{\prime}$ to

$$
u=f(x y z) \text { is }\left(x-x^{\prime}\right) \frac{d u^{\prime}}{d x^{\prime}}+\left(y-y^{\prime}\right) \frac{d u^{\prime}}{d y^{\prime}}+\left(z-z^{\prime}\right) \frac{d u^{\prime}}{d z^{\prime}}=0 .
$$

and the tangent plane at $x^{\prime} y^{\prime} z^{\prime}$ to

$$
v=f^{\prime}(x y z) \text { is }\left(x-x^{\prime}\right) \frac{d v^{\prime}}{d x^{\prime}}+\left(y-y^{\prime}\right) \frac{d v^{\prime}}{d y^{\prime}}+\left(z-z^{\prime}\right) \frac{d v^{\prime}}{d z^{\prime}}=0 .
$$

These planes are coincident if $x^{\prime} y^{\prime} z^{\prime}$ satisfies $u=o$ and $v=o$, and also

$$
d u^{\prime}=\frac{d v^{\prime}}{d x^{\prime}} ; \frac{d u^{\prime}}{d y^{\prime}}=\frac{d v^{\prime}}{d y^{\prime}} ; \quad \frac{d u^{\prime}}{d z^{\prime}}=\frac{d v^{\prime}}{d z^{\prime \prime}}
$$

In the present case $x=r \sin \theta, y=r \cos \theta$, so that

$$
\begin{aligned}
& \frac{d u}{d x}=\sin \theta \frac{d u}{d r}+\frac{\cos \theta}{r} \cdot \frac{d u}{d \theta^{\circ}} \\
& \frac{d u}{d y}=\cos \theta \frac{d u}{d r}-\frac{\sin \theta}{r} \cdot \frac{d u}{d \theta^{\circ}}
\end{aligned}
$$

Hence if $U, V$ meet at $r^{\prime} \theta^{\prime} z^{\prime}$, and have a common tangent plane there, one must have

$$
\begin{aligned}
& z^{\prime}-\frac{p \theta^{\prime}}{2 \pi}-r^{\prime} \tan \alpha=o . \\
& z^{\prime}-z_{-1}-r^{\prime} \sin \theta^{\prime} \tan \beta-\sec \beta \sqrt{c^{2}-\left(r_{o}-r^{\prime} \cos \theta^{\prime}\right)^{2}}=o . \\
& \cos \theta^{\prime} \tan \alpha-\sin \theta^{\prime} \times \frac{p}{2 \pi r^{\prime}}=\frac{\sec \beta\left(r_{0}-r^{\prime} \cos \theta^{\prime}\right)}{\sqrt{c^{2}-\left(r_{o}-r^{\prime} \cos \theta^{\prime}\right)^{2}}} \\
& \sin \theta^{\prime} \tan \alpha+\cos \theta^{\prime} \times \frac{p}{2 \pi r}=\tan \beta .
\end{aligned}
$$


The eliminant of $z^{\prime} r^{\prime} \theta^{\prime}$ from these four equations is the condition that the surfaces touch.

For convenience omit the dashes, and write $t=\tan \alpha, 8=\tan \beta$, $\mu=\frac{p}{2 \pi r^{\prime}}$. Then if $z$ be eliminated from the first two equations, it is found that

$$
\begin{aligned}
& \left.\mu r \theta+r t-k-r s \sin \theta=\sqrt{1+r^{2}} \times \sqrt{c^{2}-\left(r_{u}-r \cos \theta\right.}\right)^{2} . \\
& (t \cos \theta-\mu \sin \theta) \sqrt{c^{2}-\left(r_{1}-r \cos \theta\right)^{2}}=\sqrt{1+s^{2} \times\left(r_{o}-r \cos \theta\right) .} \\
& t \sin \theta+\mu \cos \theta=8 .
\end{aligned}
$$

From these three equations $r$ and $\theta$ have to be eliminated. They are equivalent to

$$
\begin{aligned}
& \{\mu r \theta+r t-k-r 8 \sin \theta\}(t \cos \theta-\mu \sin \theta)=\left(1+s^{2}\right)\left(r_{o}-r \cos \theta\right) . \\
& \left(r_{o}-r \cos \theta\right)\left\{(t \cos \theta-\mu \sin \theta)^{2}+1+s^{2}\right\}^{\frac{1}{2}}=c(t \cos \theta-\mu \sin \theta) . \\
& t \sin \theta+\mu \cos \theta=8 .
\end{aligned}
$$

Or, again,

$$
\begin{aligned}
& \{\mu r \theta+r t-k-r s \sin \theta\} \sqrt{t^{2}-s^{2}+} \overline{\mu^{2}}=\left(1+s^{2}\right)\left(r_{t}-r \cos \theta\right) . \\
& \left(r_{1}-r \cos \theta\right) \sqrt{ } 1^{-}+t^{2}+\mu^{2}=c \sqrt{t^{2}-8^{2}+\mu^{2} .}
\end{aligned}
$$

$t \sin \theta+\mu \cos \theta=8$.

Or,

$$
\begin{aligned}
& \{\mu r \theta+r t-k-r s \sin \theta\} \sqrt{1+t^{2}+\mu^{2}}=c\left(1+s^{2}\right) . \\
& \left(r_{0}-r \cos \theta\right) \sqrt{1+t^{2}+\mu^{2}}=e \sqrt{t^{2}-s^{2}+\mu^{2}} .
\end{aligned}
$$$$
t \sin \theta+\mu \cos \theta=8 \text {. }
$$

Eliminating $r$ from the first two of these equations

$$
\sec \theta\{t+\mu \theta-s \sin \theta\}\left\{r_{n}-c \sqrt{\frac{t^{2}-\overline{s^{2}+\mu^{2}}}{1+t^{2}+\mu^{2}}}\right\}=k+\frac{c\left(1+s^{2}\right)}{\sqrt{1+t^{2}+\mu^{2}}} .
$$

$t \sin \theta+\mu \cos \theta=8$.

from which it remains to eliminate $\theta$.

Observing that $\mu$ is small and so also is $\theta$, powers of each higher than the second may be neglected.

Accordingly the following are derived

$$
\begin{gathered}
\left(1+\frac{1}{2} \theta^{2}\right)(t+\mu \theta-s \theta)\left[v_{0}-\frac{c^{2} \sqrt{t^{2}-s^{2}}}{\sqrt{1}+t^{2}}\left\{1+\frac{\mu^{2}\left(1+s^{2}\right)}{2\left(1+t^{2}\right)\left(t^{2}-s^{2}\right)}\right\}\right] \\
=k+\frac{c\left(1+s^{2}\right)}{\sqrt{1+t^{2}}}\left\{1-\overline{2\left(1+t^{2}\right)}\right\} .
\end{gathered}
$$

$t \theta+\mu=8$. 
Hence

$$
r_{u} t=k\left\{1+\frac{(8-\mu)^{2}}{2 t^{2}}\right\}
$$

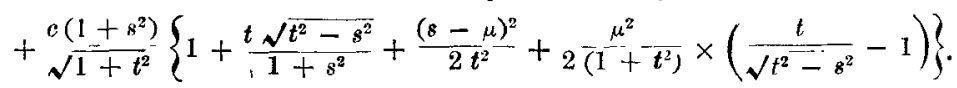

Also

$$
\begin{gathered}
\frac{1}{2} T=r_{o}+c, \text { and } k=R^{\prime} t \\
\therefore T=2 R^{\prime}\left\{1+\frac{(s-\mu)^{2}}{2 t^{2}}\right\}+2 c \\
+\frac{2 c\left(1+s^{2}\right)}{t \sqrt{1+t^{2}}}\left\{1+\frac{t \sqrt{t^{2}-s^{2}}}{1+s^{2}}+\frac{(s-\mu)^{2}}{2 t^{2}}+\frac{\mu^{2}}{2\left(1+t^{2}\right)}\left(\frac{t}{\sqrt{t^{2}-s^{2}}}-1\right)\right\} .
\end{gathered}
$$

In the cases to be dealt with $s$ is small; if the powers of $s$ higher than the second are negligible, the formula becomes

$$
\begin{gathered}
T=2 R^{\prime}\left\{1+\frac{(s-\mu)^{2}}{2 t^{2}}\right\} \\
+2 c\left\{1+\frac{\sqrt{1+t^{2}}}{t}+\frac{8^{2}}{2 t \sqrt{1+t^{2}}}+\frac{(s-\mu)^{2}}{2 t^{3} \sqrt{1+t^{2}}}\right\}
\end{gathered}
$$

Further,

$$
\begin{gathered}
2 R^{\prime}=2 P-\frac{p}{2 t} \\
\therefore T=\left(2 P-\frac{p}{2 t}\right)\left\{1+\frac{(s-\mu)^{2}}{2 t^{2}}\right\} \\
+2 c\left\{1+\frac{\sqrt{1+t^{2}}}{t}+\frac{s^{2}}{2 t \sqrt{ } 1+t^{2}}+\frac{(s-\mu)^{2}}{2 t^{3} \sqrt{ } 1+t^{2}}\right\} .
\end{gathered}
$$

This equation connects the distance over cylinders $T$ with the effective diameter, pitch, diameter of cylinder, angle of thread, rake, and angle at which the cylinder is set.

A particular case is that treated on page 1091, which may be derived from the above by writing $s=\mu$. It then becomes

$$
T=2 P-\frac{p}{2 t}+2 e\left\{1+\frac{\sqrt{1+}+t^{2}}{t}+\frac{\mu^{2}}{2 t \sqrt{1}+t^{2}}\right\},
$$

which is equivalent to the formula given (page 1091).

Another particular case of practical interest is when the cylinders are fixed perpendicular to the axial plane of the screw. 
Then $s=0$, and the formula reduces to

$$
\begin{gathered}
T=\left(2 P-\frac{p}{2 t}\right)\left(1+\frac{\mu^{2}}{2 t^{2}}\right)+2 e\left\{1+\frac{\sqrt{1+t^{2}}}{t}+\frac{\mu^{2}}{2 t^{3} \sqrt{1+t^{2}}}\right\} ; \\
\quad \text { or, } \\
T=\left(2 P-\frac{1}{2} p \cot \alpha\right)\left(1+\frac{1}{2} \mu^{2} \cot ^{2} \alpha\right)+2 c\left\{1+\operatorname{cosec} \alpha+\frac{1}{2} \mu^{2} \cos \alpha \cot ^{3} \alpha\right\} .
\end{gathered}
$$

Again, the value of $s$ may be determined so that $T$ is a minimum. Putting $\frac{d T}{d s}=o$, gives

$$
s=\mu \times \frac{R^{\prime} t \sqrt{1+t^{2}}+c}{R^{\prime} t \sqrt{1+t^{2}+o\left(1+t^{2}\right)}} .
$$

$=\mu$ nearly, since $R^{\prime}$ is large compared with $c$ and $t$ is usually about $\frac{1}{2}$. This then is the particular case already dealt with.

Limiting sizes of cylinders for measuring effective diameter of rounded threads.-To determine the limiting sizes of cylinders to be used in measuring the effective diameter of a screw.

Plainly the cylinders must not be so small that $T$ is less than the full diameter, nor so large that they do not touch the slopes of the thread.

Let $c_{1}$ be the greatest permissible value of the radius of the cylinder. Let $c_{2}$ be the least permissible radius. Let $m$ be the fraction of depth of thread that is rounded off the top, and also off the bottom, of the complete triangle. (See Fig. 17, page 1090.)

Then

$$
\begin{aligned}
& \rho(\operatorname{cosec} a-1)=\frac{1}{2} m p \cot \alpha . \\
& c_{1}=\frac{1}{2} p \sec \alpha-\rho . \\
\therefore \quad & c_{1}=\frac{1}{2} p\left\{\sec \alpha-\frac{m \cos \alpha}{1-\sin a}\right\} .
\end{aligned}
$$

This is the maximum value of the radius of cylinder permissible It has been assumed that the section of cylinder in the axial plane 0 : the screw is sufficiently nearly circular for this investigation.

The condition usually governing the lower limit of radius $i$ that

$$
T=\text { full diameter }=2 R-m p \cot \alpha
$$


It has been seen in page 1089 that

$$
\begin{gathered}
T=2 R^{\prime}+2 c_{2}(1+\operatorname{cosec} \alpha) . \\
\therefore \quad R-R^{\prime}=c_{2}(1+\operatorname{cosec} \alpha)+\frac{1}{2} m p \cot \alpha .
\end{gathered}
$$

Also

$$
\begin{gathered}
R-R^{\prime}=\frac{1}{2} p \cot \alpha . \\
\therefore \quad c_{2}=\frac{1}{2} p \times \frac{(1-m) \cos \alpha}{1+\sin \alpha} .
\end{gathered}
$$

Sometimes the lower limit may be determined by the fact that the cylinder must not rest on the rounded part of the root of the thread.

This limiting value is $c_{2}^{\prime}=\rho=\frac{m p \cos \alpha}{2(1-\sin \alpha)}$.

The greater of the values $c_{2}$ or $c_{2}^{\prime}$ is to be taken.

The criterion which determines the choice of $c_{2}$ is that $c_{e_{2}^{\prime}}>1$; i.e., $\frac{(1-m)(1-\sin \alpha)}{m(1+\sin \alpha)}>1$; or $\sin \alpha<1-2 m$.

Thus $c_{2}$ is used if $\sin \alpha<1-2 m$, and $c_{2}^{\prime}$ is used if sin $a>$ $1-2 \mathrm{~m}$.

This is for rounded threads.

Limiting sizes of cylinders for measuring effective diameter of trincated threads.-For truncated threads if we work similarly we find, if $m$ be the fraction of depth of thread that is eut off both top and bottom of the complete triangle by a line parallel to the axis,

$$
\begin{aligned}
& c_{1}=\frac{1}{2} p(1-m) \cdot \sec \alpha . \\
& c_{2}=\frac{1}{2} p \frac{(1-m)}{1+\sin \alpha} . \\
& c_{2}^{\prime}=\frac{m p \cos \alpha}{2(1-\sin \alpha)} .
\end{aligned}
$$

$c_{2}^{\prime}$ being used instead of $c_{2}$ if $\sin a>1-2 \mathrm{~m}$.

The radius of mean cylinder (tonching thread at mid slope) for rounded or truncated threads is $\frac{1}{4} p$ sec $\alpha$.

The effective diameter which is usually verified by measurement is approximately a line whose intercept by the surface is bisected perpendicularly by the axis of the screw. 
Limiting sizes of cylinders for Whitworth threads.-Some of the preceding results will now be applied to the case of British Standard Whitworth throads.

For measurement of effective diameter the cylinder diameters are:-

$$
\begin{aligned}
& \text { Maximum permissible cylinder diameter }=p \times 0.8527238 \text {. } \\
& \text { Minimum " } \quad "=p \times 0.5056791 \text {. } \\
& \text { Mean cylinder diameter } \quad=p \times 0.5636910 \text {. }
\end{aligned}
$$

The depth of thread is $\frac{1}{2}(1-2 m) p \cot a=p \times 0.6403274$. Thus the mean eylinder diameter equals the depth of thread $\times 0.88032$.

Formula for effective diameter of Whitworth threads.-The formula for effective diameter may be adapted for Whitworth threads as follows :-

The formula is

$$
2 P=T+\frac{1}{2} p \cot \alpha-2 \circ\left\{1+\operatorname{cosec} \alpha \sqrt{1+\mu^{2} \cos ^{2} \alpha}\right\} .
$$

Now approximately

$$
\sqrt{1+\mu^{2} \cos ^{2} \alpha}=1+\frac{1}{2} \mu^{2} \cos ^{2} \alpha,
$$

so that

$$
\begin{aligned}
& T=2 P-\frac{1}{2} p \cot \alpha+2 c\left\{1+\operatorname{cosec} \alpha+\frac{1}{2} \mu^{2} \cos \alpha \cot \alpha\right\} . \\
& \text { Put } \alpha=27 \frac{10}{2} \text {. } \\
& \therefore \quad T=2 P-p \times 0 \cdot 9604910+2 c\left\{3 \cdot 1656806+\mu^{2} \times 0 \cdot 8519660\right\} . \\
& \mu=\frac{p}{2 \pi r} \text {, so that } \mu^{2} \text { is small; and if the value of the cylinder } \\
& \text { diameter be not far from that of the mean cylinder we may write } P \\
& \text { for } r \text { in this expression. Thus } \mu=\frac{p}{2 \pi P} .
\end{aligned}
$$$$
\text { Accordingly } \mu^{2}=0.1013212 \times\left(\begin{array}{c}
p \\
2 \bar{P}
\end{array}\right)^{2} .
$$$$
\therefore \quad T=2 P-p \times 0.9604910+2 o\left(\begin{array}{c}
p \\
2 F^{\prime}
\end{array}\right)^{2} \times 0.0863222+2 c \times 3 \cdot 1656806 .
$$

The third term on the right hand side is small, so we may use the mean cylinder diameter for $2 c$ in it, and thus obtain 


$$
\begin{aligned}
T= & 2 P-p \times 0.9604910+p \times\left(\frac{p}{2 P}\right)^{2} \times 0.0486590+2 c \times 3 \cdot 1656806 . \\
= & A+2 c \times 3 \cdot 1656806 . \\
& \text { where } A=2 P-p \times 09604910+p \times\left(\frac{p}{2 P}\right)^{2} \times 0.0486590 .
\end{aligned}
$$

Later, the various values of $A$ for the different sizes of screws will be tabulated for British Standard Whitworth, and Fine, threads.

Also will be given the values of $T$ obtained by taking for $2 c$ the mean cylinder diameter in each case. These values of $T$ will be based on all the elements of the screw being correct.

Effect of errors in various elements on effective diameter as measured.--If there is an error in the effective diameter only, that error is equivalent to the observed error in $T$. But in general there are errors in all the elements, and the effective diameter (as measured) is connected with the other elements by the relation

$$
2 P=T+\frac{1}{2} p \cot \alpha-2 c\left\{1+\operatorname{cosec} \alpha \sqrt{1+\mu^{2} \overline{\cos ^{2} \alpha}}\right\} \text {. }
$$

Let there be errors in $a, p, T, \mu, c$ of amounts $\delta a, \delta p, \delta T$, $\delta \mu, \delta c$.

Then the error in effective diameter $\delta(2 P)$ is

$\delta(2 P)=\delta T+\frac{1}{2} \cot \alpha \delta p-\delta(2 c) \times\left\{1+\operatorname{cosec} a \sqrt{1+\mu^{3} \cos ^{2}} a\right\}$

$$
\begin{aligned}
& +\delta \alpha \times\left\{\frac{2 e\left(1+\mu^{2}\right) \operatorname{cosec} \alpha \cot \alpha}{\sqrt{1+\mu^{2} \cos ^{2} \alpha}-\frac{1}{2} p \operatorname{cosec}^{2} \alpha}\right\} \\
& -\delta \mu \times \frac{2 c \mu \cos \alpha \cot \alpha}{\sqrt{1}+\mu^{2} \cos ^{2} \alpha} .
\end{aligned}
$$

$=\delta T+\frac{1}{2} \cot a \cdot \delta p-\delta(2 c) \times\left\{1+\operatorname{cosec} a+\frac{1}{2} \mu^{2} \cos \alpha \cdot \cot a\right\}$

$+\delta a \times\left\{2 c \operatorname{cosec} \alpha \cot \alpha+c \mu^{2} \cot \alpha(\sin \alpha+\operatorname{cosec} \alpha)-\frac{1}{2} p \operatorname{cosec}^{2} a\right\}$

$-\delta \mu \times 2 c \mu \cos \alpha \cot \alpha\left(1-\frac{1}{2} \mu^{2} \cos ^{2} \alpha\right)$. A pproximatcly.

The mean cylinder is supposed used (in the case of a correct screw), one has so $\mu=\frac{p}{2 \pi P}$; and $\delta \mu=\frac{1}{2 \pi}\left\{\frac{\delta p}{P^{\prime}}-\frac{p \delta p}{p^{2}}\right\}$.

Thus one finds

$$
\begin{aligned}
& \delta\left(2 P ; \times\left\{1-\frac{c p^{2}}{2 \pi^{2} P^{3}} \cos \alpha \cot \alpha\left(1-\frac{p^{2} \cos ^{2} \alpha}{8 \pi^{2} P^{2}}\right)\right\}\right. \\
& =\delta T+\delta p \times\left\{\frac{1}{2} \cot \alpha-\frac{c p}{2 \pi^{2} P^{2}} \cos \alpha \cot \alpha\left(1-\frac{p^{2} \cos ^{2} \alpha}{\overline{8} \pi^{2} \bar{P}^{2}}\right)\right\}
\end{aligned}
$$


$+\delta \alpha \times\left\{2 c \operatorname{cosec} \alpha \cot \alpha+\frac{c p^{2}}{8 \pi^{2}} \bar{p}^{2} \cot \alpha(\sin \alpha+\operatorname{cosec} \alpha)-\frac{1}{2} p \operatorname{cosec}^{2} \alpha\right\}$

$$
-\delta(2 c)\left\{1+\operatorname{cosec} \alpha+\frac{p^{2}}{8 \pi^{2} P^{2}} \cos \alpha \cot \alpha\right\} \text {. }
$$

Again, omitting small quantities the following is obtained

$$
\begin{gathered}
\delta(2 P)=\delta T+\delta p \times \frac{1}{2} \cot a+\delta a \times\left\{2 c \operatorname{cosec} a \cot a-\frac{1}{2} p \operatorname{cosec}^{2} \alpha\right\} \\
-\delta(2 c) \times(1+\operatorname{cosec} \alpha),
\end{gathered}
$$

or

$$
\delta(2 P)=\delta T+0 \cdot 9604910 \delta p-3 \cdot 1656806 \delta(2 o) \text {. }
$$

The coefficient of $\delta$ a vanishes since $2 c=\frac{1}{2} p$ sec $a$. In words

Error in effective diameter $=$ error in $T+0.96 \times$ error in pitch $-3 \cdot 17 \times$ error in cylinder diameter.

The error in $T$ is the difference between the measured value and that for a correct screw (using the mean cylinder). The correct cylinder touches the slopes of a correct screw midway on the slope.

If the cylinder used has a diameter differing from the mean cylinder diameter for the size in question by more than 0.001 inch, we use

$$
A=T-2 c \times 3 \cdot 1656806
$$

where $2 c$ is the diameter of the cylinder used, and $T$ is the measured thickness over oylinders.

In this case the effect of errors in other elements of the screw on effective diameter is

$$
\delta \cdot(2 P)=\delta A+\delta p \times \frac{1}{2} \cot \alpha+\delta a \times\left\{2 o \operatorname{cosec} \alpha \cot a-\frac{1}{2} p \operatorname{cosec}^{2} a\right\} ;
$$

where $\delta A$ is the difference between the values of $A$ for a correct screw, and that got from measurements of $T$ and $2 e$ above on the screw in question.

If the numerical constants for Whitworth threads be supplied, and the error in angle be expressed in degrees the following is obtained

$$
\begin{gathered}
\delta(2 P)=\delta A+0.9604910 \times \delta p \\
+\delta a^{3} \times\{2 c \times 0.0726097-p \times 0.0409294\} .
\end{gathered}
$$

$\mathrm{Or}$, in words

Error in effective diameter $=$ error in $A+0.96 \times$ error in pitch + trror in full angle in degrees $\times\{2 e \times 0.036-p \times 0.020\}$. 
The correct values of $T$ and $A$, and the mean cylinder diameter, are given in the Tables for British Standard Whitworth, and Fine, threads (pages 1107 and 1108).

Effective diameter of Whitworth thread with cylinders perpendicular to axis of screw.-The formula for effective diameter, whon the cylinders are held perpendicular to the axial plane of measurement of the screw, has been given in page 1095, viz. :-

$$
\begin{gathered}
T=2 P-\frac{1}{2} p \cot \alpha+2 c(1+\operatorname{cosec} \alpha) \\
+\frac{1}{2} \mu^{2} \cot ^{2} \alpha\left(2 P-\frac{1}{2} p \cot \alpha+2 c \cos \alpha \cot a\right) .
\end{gathered}
$$

To adapt this for Whitworth threads we put $2 a=55^{\circ}$, and obtain

$$
\begin{gathered}
T=2 P-p \times 0 \cdot 9604910+2 c \times 3 \cdot 1656806 \\
+\mu^{2} \times 1 \cdot 8450861 \times\{2 P-p \times 0 \cdot 9604910+2 c \times 1 \cdot 7039319\} .
\end{gathered}
$$

where $\mu$ stands for $\frac{p}{2 \pi r}$, as before.

Nature of shadow of a screw in parallel light.-If a beam of parallel light fall on a screw, it might at first be supposed that the shadow is an exact representation of an axial plane section of the screw. This, however, is not quite the case.

Let parallel beam of light fall on a screw in a direction making an angle $\frac{\pi}{2}+\beta$ with the axis of the screw, and let the shadow be cast on a plane perpendicular to the direction of the light.

Let rectangular axes of $x$ and $y$ be taken in this plane, the axis of $x$ coinciding with the projection of the axis of the screw, the equation to the bounding curve of the shadow may then be determined, and for convenience the thread is taken as being purely triangular. Part of the surface of the screw is represented by the equation

$$
z-\frac{p \theta}{2 \pi}=r \tan \alpha
$$

Also

$$
\tilde{t} \sec \beta=z-r \tan \beta \sin \left(\theta-\theta_{o}\right)
$$


$y=r \cos \left(\theta-\theta_{o}\right)$, where $\theta_{o}$ corresponds to the values of $r$ whioh are perpendicular to the beam of light. Eliminating $z$ we obtain

$$
r\left\{\tan \alpha-\tan \beta \sin \left(\theta-\theta_{0}\right)\right\}=x \sec \beta-\frac{p \theta}{2 \pi} ;
$$

and eliminating $r$ between this and the equation for $y$ we got

$$
y=\frac{\left(x \sec \beta-\frac{p \theta}{2 \pi}\right) \cdot \cos \left(\theta-\theta_{0}\right)}{\tan \alpha-\tan \beta \sin \left(\theta-\theta_{o}\right)} .
$$

This equation connects the $x$ and $y$ co-ordinates of the projection of any point on the part of the screw considered. The bounding curve of this shadow will be obtained by choosing the maximum value of $y$ for each value of $x$. Hence $\frac{d y}{d \theta}=0$, an equation which determines the value of $\theta$ for which $y$ is a maximum corresponding to any given value of $x$. Fliminating $\theta$ between this and the above equation for $y$, a relation between $x$ and $y$ is obtained, in other words, the equation to the part of the bounding curve in question.

$$
\begin{aligned}
& \frac{d y}{d \theta}= \frac{-\left(x \sec \beta-\frac{p \theta}{2 \pi}\right) \sin \left(\theta-\theta_{0}\right)-\frac{p}{2 \pi} \cos \left(\theta-\theta_{0}\right)}{\tan \alpha-\tan \beta \sin \left(\theta-\theta_{0}\right)} \\
&+\frac{\left(x \sec \beta-\frac{p \theta}{2 \pi}\right) \cos ^{2}\left(\theta-\theta_{0}\right) \tan \beta}{\left\{\tan \alpha-\tan \beta \sin \left(\theta-\theta_{0}\right)\right\}^{2}} . \\
& \therefore\left\{\left(x \sec \beta-\frac{p \theta}{2 \pi}\right) \sin \left(\theta-\theta_{0}\right)+\frac{p}{2 \pi} \cos \left(\theta-\theta_{0}\right)\right\}\left\{\tan \alpha-\tan \beta \sin \left(\theta-\theta_{0}\right)\right\} \\
&-\tan \beta \cos ^{2}\left(\theta-\theta_{0}\right) \cdot\left(x \sec \beta-\frac{p \theta}{2 \pi}\right)=0 . \\
& \quad\left(x \sec \beta-\frac{p \theta}{2 \pi}\right)\left\{\tan \alpha \cdot \sin \left(\theta-\theta_{0}\right)-\tan \beta\right\} \\
&+\frac{p}{2 \pi} \cos \left(\theta-\theta_{o}\right)\left\{\tan \alpha-\tan \beta \cdot \sin \left(\theta-\theta_{0}\right)\right\}=0 .
\end{aligned}
$$

Now

$$
\begin{gathered}
\left(x \sec \beta-\frac{p \theta}{2 \pi}\right) \cos \left(\theta-\theta_{0}\right)=y\left\{\tan \alpha-\tan \beta \sin \left(\theta-\theta_{0}\right)\right\} . \\
\therefore y\left\{\tan \alpha \sin \left(\theta-\theta_{0}\right)-\tan \beta\right\}+\frac{p}{2} \frac{p}{\pi} \cos ^{2}\left(\theta-\theta_{0}\right)=0,
\end{gathered}
$$

or

$$
\tan a-\tan \beta \sin \left(\theta-\theta_{o}\right)=o \text {. }
$$


Reject the latter equation as it requires $\beta>\alpha$.

Hence

$$
\begin{aligned}
& \sin ^{2}\left(\theta-\theta_{0}\right)-\frac{2 \pi y}{p} \tan \alpha \sin \left(\theta-\theta_{o}\right)+\frac{2 \pi y}{p} \tan \beta-1=0 . \\
\therefore & \sin \left(\theta-\theta_{0}\right)=\frac{\pi y}{p} \tan a-\sqrt{\frac{\pi^{2} y^{2}}{p^{2}} \tan ^{2} \alpha-\frac{2 \pi y}{p} \tan \beta+1 .}
\end{aligned}
$$

$=M$ say, in which the negative sign for the root is chosen, as $\pi y \tan a$ may be taken to be always greater than $p$, in the part considered.

$\therefore y(\tan \alpha-M \tan \beta)=\left\{x \sec \beta-\frac{p}{2} \frac{p}{\pi}\left(\theta_{o}+\sin ^{-1} M\right)\right\} \sqrt{1-} M^{2}$.

This is the equation to the bounding curve of the part of the shadow in question; and $\theta_{o}$ may be taken as $0,2 \pi, 4 \pi$, etc., successively to get each of the slopes.

The remaining part of the shadow may be determined similarly, but using for the surface equation

$$
z-\frac{p \theta}{2 \pi}=\left(2 R^{\prime}-r\right) \tan \alpha .
$$

This gives

$$
\begin{gathered}
\left(\tan a+M^{\prime} \tan \beta\right)= \\
\left\{-x \sec \beta+2 \lambda^{\prime} \tan \alpha+\frac{p}{2} \frac{p}{\pi}\left(\theta_{1}+\sin ^{-1} M^{\prime}\right)\right\} \sqrt{ } 1-M^{\prime 2} .
\end{gathered}
$$

where

$$
M^{\prime}=-\frac{\pi y}{p} \tan \alpha+\sqrt{\frac{\pi^{2} y^{2}}{p^{2}} \tan ^{2} \alpha-\frac{2 \pi y}{p} \tan \beta+1}=-M ;
$$

and $\theta_{o}$ is to be taken as $\pi, 3 \pi$, etc., successively to get the various slopes.

Numerical Example.-The following figures illustrate very roughly the differences between the dimensions of an axial plane section of a screw and the corresponding dimensions of the shadow. The screw is supposed to have an effective diameter of 1 inch, a pitch of 0.125 inch, angle of $55^{\circ}$, and to have a purely triangular or $V$ thread. 
I. Direction of light perpendicular to axis of screw (i.e., $\beta=0$ ). Angle of space between threads (in the shadow) varies from about $53 \cdot 2^{\circ}$ at root to $56 \cdot 8^{\circ}$ at tip.

Effective diameter of shadow is about 0.0032 inch greater than the effective diameter of screw.

Axial length of space between threads at half the height of the thread of the shadow $=\frac{1}{2}$ pitch of screw -0.0016 inch.

Axiel length of thread (thickness) at half the height of the thread of the shadow $=\frac{1}{2}$ pitch of screw +0.0016 inch.

Pitch of shadow = Pitch of screw.

II. Direction of light along the rake of thread at half its height (i.e., $\beta=$ angle of rake of thread at half its height). Angle of space between threads, at a distance from the axis slightly less than the effective radius is about $55 \cdot 05^{\circ}$, and at a distance slightly greater is about $54 \cdot 95^{\circ}$.

The radius of the screw to $\frac{3}{4}$ height of thread is exceeded in the shadow by about 0.000035 inch.

The pitch of the screw exceeds the pitch of the shadow by about 0.000088 inch per pitch.

Axial length of space between threads at $\frac{3}{4}$ height of thread in the shadow $=\frac{3}{4}$ pitch of screw -0.000100 inoh $=\frac{3}{4}$ pitch of shadow -0.000034 inch.

Axial length of thread (thickness) at $\frac{3}{4}$ height of thread in the shadow $=\frac{1}{4}$ pitch of screw +0.000012 inch $=\frac{1}{4}$ pitch of shadow +0.000034 inch.

It will be noticed here that while one side of the screw gives a good shadow, the other side gives a bad one, since a screw rakes in different directions on opposite sides.

Effect of tilt of cutting-tool on the thread derived.-When accurate results are required, screw threads are cut in the lathe with a cutting tool of appropriate shape, and the tool is set so that the cutting edges lie as nearly as possible in a plane containing the axis of the screw. In consequence of the difficulty of setting the tool edges in the axial plane, the thread derived will not be precisely that represented by the tool edge. How far inaccuracy in thread is introduced by 
inaccuracy in setting will be understood by consideration of the case of a $V$ thread. For simplicity only one side of the thread will be dealt with-the tool edge being a simple straight line-and it will be obvious that similar results may be obtained for the other edge of the tool.

Following the notation and method in page 1071, the equations of the generating line of the surface (or tool edge) may be written in the form

$$
z-\zeta=\sqrt{r^{2}-v^{2}} \cdot \tan \alpha,
$$

and

$$
r \cos (\theta-\phi)=v
$$

where $v$ is the shortest distance between the tool edge direction and the axis of the screw; $a$ is the angle which the projection of this generating line on a parallel plane containing the axis makes with a perpendicular to the axis; $\phi$ is the angular co-ordinate of the shortest distance $v$, reckoned like $\theta$ round the axis of $z$; and $\theta$ is the ordinary angular co-ordinate of any point on the generating line.

Now as this line swings round the axis of the screw, let it at the same time travel forward parallel to the axis, and a surface is swept out. This generated surface is half the surface of the screw thread.

Select the origin of $z$ so that $z=0$ when $r=v$ and $\phi+\frac{\pi}{2}=0$. Hence $\zeta=\frac{p}{2 \pi}\left(\phi+\frac{\pi}{2}\right)$. Then the surface generated is represented by

$$
z-\frac{p}{4}-\frac{p \phi}{2 \pi}=\sqrt{r^{2}-v^{2}} \cdot \tan a ;
$$

and since $r \cos (\theta-\phi)=v$, the equation to the surface is written

$$
\left.\right|_{r=n} ^{r=R^{r}} \approx-\frac{p}{4}-\frac{p}{2 \pi}\left(\theta-\cos ^{-1} \cdot \frac{v}{r}\right)=\sqrt{r^{2}-v^{2}} \cdot \tan \alpha .
$$

In like manner the equation to the other portion of the surface may be obtained. 
Note that, if $v=0, \phi=\theta-\frac{\pi}{2}$ and the equation becomes

$$
\left.\right|_{r=R} ^{r=R^{\prime}} z-\frac{p \theta}{2 \pi}=r \tan \alpha,
$$

as on page 1071 .

Having thus obtained the equation to the surface, it is easy to write down the equation of an axial plane section, for one has only to equate $\theta$ to a constant, and for convenience this constant may be taken equal to $o$. Thus the equation of curre of one face of thread in an axial plane is

$$
z-\frac{p}{4}+{ }_{2 \pi}^{p} \cdot \cos _{r}^{-1}{ }_{r}^{n}=\sqrt{r^{2}-v^{2}} \cdot \tan \alpha .
$$

For the parts of the thread considered $v$ is usually small compared with $r$, so that powers of $\frac{v}{r}$ higher than the second or third may be neglected.

Now

$$
\operatorname{cns}^{-1}{ }_{r}^{v}=\frac{\pi}{2}-\frac{n}{r}-\frac{1}{2} \cdot \frac{v^{3}}{3 r^{3}}-\frac{1 \cdot 3}{2 \cdot 4} \cdot \frac{v^{5}}{5}-\frac{1}{r^{3}}-\ldots
$$

if $\frac{v}{r}$ lies between $a$ an 1 .

Hence approximately

$$
\cos ^{-1} \frac{v}{r}=\frac{\pi}{2}-\frac{v}{r}-\frac{n^{3}}{6 r^{3}}
$$

The axial plane section then becomes

$$
\begin{gathered}
z-\frac{p}{2 \pi}\left(\frac{v}{r}+\frac{v^{3}}{6 r^{3}}\right)=\sqrt{r^{2}-1 v^{2}} \cdot \tan a \\
=r \tan a\left(1-\frac{v^{2}}{2 r^{2}}\right) \text { approximately; } \\
z=r \tan \alpha+\frac{v}{2 r}\left\{\frac{p}{\pi}+\frac{p v^{2}}{6 \pi r^{2}}-v \tan a\right\} .
\end{gathered}
$$

or

If $v=o$ this becomes $z=r \tan \alpha$, as on page 1071 .

Thus the departure in the axial direction from the straight line $z=r$ tan $a$ at a point distant $r$ from the axis, is given by

$$
\frac{v}{2 r}\left\{\frac{p}{\pi}+\frac{p v^{2}}{6 \pi r^{2}}-v \tan a\right\}
$$


This is greatest (for a given arithmetical value of $v$ ) when $v$ is negative, and the departure then is

$$
-\frac{v}{2 r}\left\{\frac{p}{\pi}+\frac{p v^{2}}{6 \pi r^{2}}+v \tan \alpha\right\}
$$

where $v$ is the arithmetical value.

Here, in fact, $\phi$ is greater than $\theta$, and $z=0$ when $\phi=\frac{\pi}{2}$, and $r=v$; so that the surface becomes

$$
z+\frac{p}{4}-\frac{p \phi}{2 \pi}=\sqrt{r^{2}-v^{2}} \cdot \tan \alpha, \text { and } r \cos (\phi-\theta)=v .
$$

Therefore the surface equation is

$$
\left.\right|_{r=R} ^{r=R^{\prime}} z+\frac{p}{4}-\frac{p}{2} \pi\left(\theta+\cos ^{-1} \frac{v}{r}\right)=\sqrt{r^{2}-v^{2}} \cdot \tan \alpha .
$$

The axial plane section $\theta=o$ then becomes

$$
\therefore=r \tan a-\frac{v}{2}\left\{\frac{p}{\pi}+\begin{array}{c}
p r^{2} \\
6 \pi r^{2}
\end{array}+v \tan a\right\} .
$$

As an illustration one may take the case of a $V$ thread having effective diameter 1 inch, pitch $0.125 \mathrm{inch}$, angle $55^{\circ}$. Let the tool edge be tilted $5^{\circ}$ from its correct position, in a plane perpendicular to the axial plane containing its middle point, and on the side of the axial plane that makes $v$ negative. Then $v=P \cdot \sec a \cdot \sin 5^{\circ} ; P=\frac{1}{2}$ inch; $a=27 \frac{1}{2}^{\circ}$. Hence $v=0.0491$ inch. Full diameter $2 R=1.12$ inch; core diameter $2 R^{\prime}=0.88 \mathrm{inch}$. Hence, substituting for $r$ the values 0.56 inch and 0.44 inch in succession, one finds the departures from the straight line $z=r \tan a$ to $b_{\theta} 0.00287$ inch and 0.00365 inch at the crest and root respectively. The difference between these departures 0.00078 inch is the amount of deviation in the axial direction between crest and root of the thread. The deviations at intermediate points on the thread may in like manner be worked out, and the axial plane section of the thread exhibited.

The Paper is illustrated by 17 Figs. in the letterpress. 
TABLE 1.-British Standard Whitworth Threads.

\begin{tabular}{|c|c|c|c|c|c|c|c|c|}
\hline 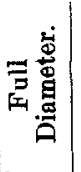 & 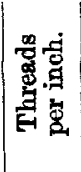 & 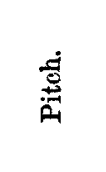 & 吾总 & 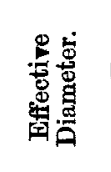 & 悹蓠 & 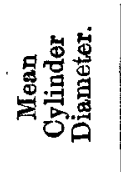 & 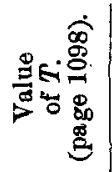 & 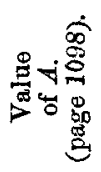 \\
\hline In. & & Inch. & Inch. & Inch. & Inch. & Inch. & Inch. & Inch. \\
\hline$\frac{1}{4}$ & 20 & 0.0500 & 0.0320 & $0 \cdot 2180$ & $0 \cdot 1860$ & 0.02818 & 0.2594 & $0 \cdot 1701$ \\
\hline$\frac{5}{18}$ & 18 & 0.0556 & 0.0356 & $0 \cdot 2769$ & 0.2414 & 0.03132 & $0 \cdot 3227$ & 0.2236 \\
\hline$\frac{8}{8}$ & 16 & 0.0625 & 0.0400 & $0 \cdot 3350$ & $0 \cdot 2950$ & $0 \cdot 03523$ & $0 \cdot 3866$ & $0 \cdot 2751$ \\
\hline$x^{7} 8$ & 14 & 0.0714 & 0.0457 & $0 \cdot 3918$ & $0 \cdot 3460$ & 0.04026 & $0 \cdot 4508$ & $0 \cdot 3233$ \\
\hline$\frac{1}{2}$ & 12 & $0 \cdot 0833$ & $0 \cdot 0533$ & $0 \cdot 4466$ & $0 \cdot 3933$ & $0 \cdot 04697$ & $0 \cdot 5154$ & $0 \cdot 3667$ \\
\hline$\frac{9}{16}$ & 12 & $0 \cdot 0839$ & 0.0533 & $0 \cdot 5091$ & $0 \cdot 4558$ & $0 \cdot 04697$ & 0.5779 & $0 \cdot 4292$ \\
\hline$\frac{5}{8}$ & 11 & $0 \cdot 0909$ & 0.0582 & 0.5668 & $0 \cdot 5086$ & $0 \cdot 05124$ & $0 \cdot 6418$ & $0 \cdot 4796$ \\
\hline$\frac{11}{6}$ & 11 & 0.0909 & 0.0582 & 0.6293 & $0 \cdot 5711$ & $0 \cdot 05124$ & 0.7043 & 0.5421 \\
\hline$\frac{3}{4}$ & 10 & $0 \cdot 1000$ & $0 \cdot 0640$ & $0 \cdot 6860$ & 0.6219 & 0.05637 & $0 \cdot 7685$ & 0.5901 \\
\hline $\begin{array}{l}13 \\
16\end{array}$ & 10 & $0 \cdot 1000$ & $0 \cdot 0640$ & $0 \cdot 7485$ & $0 \cdot 6844$ & $0 \cdot 05637$ & $0 \cdot 8310$ & 0.6526 \\
\hline$\frac{7}{8}$ & 9 & $0 \cdot 1111$ & 0.0711 & 0.8039 & $0 \cdot 7327$ & 0.06263 & 0.8956 & 0.6973 \\
\hline 1 & 8 & $0 \cdot 1250$ & 0.0800 & $0 \cdot 9200$ & 0.8399 & $0 \cdot 07046$ & $1 \cdot 0231$ & 0.8000 \\
\hline $1 \frac{1}{8}$ & 7 & $0 \cdot 1429$ & $0 \cdot 0915$ & $1 \cdot 0335$ & 0.9420 & 0.08053 & $1 \cdot 1514$ & 0.8964 \\
\hline 17 & 7 & $0 \cdot 1429$ & $0 \cdot 0915$ & $1 \cdot 1585$ & $1 \cdot 0670$ & $0 \cdot 08053$ & $1 \cdot 2763$ & $1 \cdot 0214$ \\
\hline $1 \frac{3}{y}$ & 6 & $0 \cdot 1667$ & $0 \cdot 1067$ & $1 \cdot 2683$ & $1 \cdot 1616$ & 0.09395 & $1 \cdot 4058$ & $1 \cdot 1083$ \\
\hline $1 \frac{7}{2}$ & 6 & $0 \cdot 1667$ & $0 \cdot 1067$ & $1 \cdot 3933$ & $1 \cdot 2866$ & 0.09395 & $1 \cdot 5307$ & $1 \cdot 2333$ \\
\hline $1 \frac{5}{8}$ & 5 & $0 \cdot 2000$ & $0 \cdot 1280$ & $1 \cdot 4969$ & $1 \cdot 3689$ & 0.11274 & $1 \cdot 6619$ & $1 \cdot 3050$ \\
\hline 13 & 5 & $0 \cdot 2000$ & $0 \cdot 1280$ & $1 \cdot 6219$ & $1 \cdot 4939$ & $0 \cdot 11274$ & $1 \cdot 7868$ & $1 \cdot 4300$ \\
\hline 2 & $4 \frac{1}{2}$ & $0 \cdot 2222$ & $0 \cdot 1423$ & $1 \cdot 8577$ & $1 \cdot 7154$ & $0 \cdot 12526$ & $2 \cdot 0410$ & $1 \cdot 6445$ \\
\hline 21 & 4 & $0 \cdot 2500$ & $0 \cdot 1601$ & $.2 \cdot 0899$ & $1 \cdot 9298$ & $0 \cdot 14092$ & $2 \cdot 2961$ & $1 \cdot 8500$ \\
\hline $2 \frac{1}{2}$ & 4 & 0.2500 & $0 \cdot 1601$ & $2 \cdot 3399$ & $2 \cdot 1798$ & $0 \cdot 14092$ & $2 \cdot 5461$ & $2 \cdot 0999$ \\
\hline $2 \frac{3}{4}$ & $3 \frac{7}{2}$ & $0 \cdot 2857$ & $0 \cdot 1829$ & $\mathbf{2} \cdot \mathbf{5 6 7 1}$ & $2 \cdot 3811$ & $0 \cdot 16105$ & $2 \cdot 8027$ & $2 \cdot 2929$ \\
\hline 3 & $3 \frac{7}{2}$ & $0 \cdot 2857$ & $0 \cdot 1829$ & $2 \cdot 8171$ & $2 \cdot 6341$ & $0 \cdot 16105$ & $3 \cdot 0527$ & $2 \cdot 5428$ \\
\hline $3 \frac{1}{4}$ & 34 & $0 \cdot 3077$ & $0 \cdot 1970$ & $3 \cdot 0530$ & $2 \cdot 8560$ & $0 \cdot 17344$ & $3 \cdot 3067$ & $2 \cdot 7577$ \\
\hline $3 \frac{1}{3}$ & 31 & $0 \cdot 3077$ & $0 \cdot 1970$ & $3 \cdot 3030$ & $3 \cdot 1060$ & $0 \cdot 17344$ & $3 \cdot 5567$ & $3 \cdot 0076$ \\
\hline 39 & 3 & $0 \cdot 3333$ & 0.2134 & $3 \cdot 5366$ & $3 \cdot 3231$ & $0 \cdot 18790$ & $3 \cdot 8114$ & $3 \cdot 2165$ \\
\hline 4 & $\mathbf{3}$ & $0 \cdot \mathbf{3 3 3 3}$ & $0 \cdot 2134$ & $3 \cdot 7866$ & $3 \cdot 5731$ & $0 \cdot 18790$ & $4 \cdot 0614$ & $3 \cdot 4665$ \\
\hline $4 \frac{7}{2}$ & 27 & $0 \cdot 3478$ & 0.2227 & $4 \cdot 2773$ & $4 \cdot 0546$ & $0 \cdot 19607$ & $4 \cdot 5640$ & $3 \cdot 9433$ \\
\hline 5 & 29 & $0 \cdot 3636$ & $0 \cdot 2328$ & $4 \cdot 7672$ & $4 \cdot 5343$ & $0 \cdot 20498$ & $5 \cdot 0669$ & $4 \cdot 4180$ \\
\hline $5 \frac{1}{2}$ & 25 & $0 \cdot 3810$ & 0.2439 & $5 \cdot 2561$ & $5 \cdot 0121$ & $0 \cdot 21474$ & $5 \cdot 5701$ & $4 \cdot 8903$ \\
\hline 6 & 21 & $0 \cdot 4000$ & $0 \cdot 2561$ & $5 \cdot 7439$ & $5 \cdot 4877$ & $0 \cdot 22548$ & 6.0736 & $5 \cdot 3598$ \\
\hline
\end{tabular}


TABLE 2.-British Standard Fine Threads.

\begin{tabular}{|c|c|c|c|c|c|c|c|c|}
\hline 离弯 & 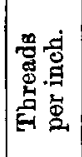 & بن & 总营 & 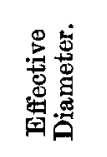 & 它 & 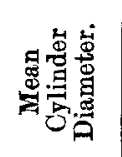 & 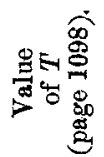 & 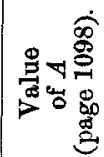 \\
\hline In. & & Inch. & Inch. & & Inch. & Inch. & Inch. & Inoh \\
\hline$\frac{1}{4}$ & 25 & $0 \cdot 0400$ & 0.0256 & $0 \cdot 2244$ & $0 \cdot 1988$ & $0 \cdot 02255$ & 0.2574 & 1861 \\
\hline$\frac{5}{16}$ & 22 & $0 \cdot 0455$ & $0 \cdot 0291$ & $0 \cdot 2834$ & 0.2543 & 0.02562 & 0.3209 & $0 \cdot 239$ \\
\hline$\frac{3}{8}$ & 20 & 0.0500 & 0.0320 & $0 \cdot 3430$ & 0.3110 & $0 \cdot 02818$ & 0.3843 & 951 \\
\hline$\frac{7}{18}$ & 18 & 0.0556 & 0.0356 & $0 \cdot 4019$ & $0 \cdot 3663$ & $0 \cdot 03132$ & 0.4477 & 3486 \\
\hline$\frac{1}{2}$ & 16 & 0.0625 & 0.0400 & $0 \cdot 4600$ & 0.4200 & $0 \cdot 03523$ & 0.5116 & .001 \\
\hline $1^{9} 8$ & 16 & 0.0625 & $0 \cdot 0400$ & $0 \cdot 5225$ & 0.4825 & $0 \cdot 03523$ & 0.5741 & 0.4626 \\
\hline 5 & 14 & 0.0714 & $0 \cdot 0457$ & 0.5793 & 0.5336 & $0 \cdot 04026$ & 0.6383 & 0.5108 \\
\hline$\frac{11}{16}$ & 14 & 0.0714 & 0.0457 & $0 \cdot 6$ & $0 \cdot 5961$ & $0 \cdot 04026$ & 0.7008 & 0.5738 \\
\hline$\frac{3}{4}$ & 12 & $0 \cdot 0833$ & 0.0534 & 0.6 & 0.6432 & $0 \cdot 04697$ & & 0.6167 \\
\hline$\frac{13}{16}$ & 12 & 0.0833 & 0.0534 & $0 \cdot 7591$ & $0 \cdot 7057$ & $0 \cdot 04697$ & 0.8279 & $0 \cdot 6792$ \\
\hline$\frac{7}{8}$ & 11 & 0.0909 & 0.0582 & 0.8168 & 0.7586 & $0 \cdot 05124$ & 0.8918 & 0.7296 \\
\hline 1 & 10 & $0 \cdot 1000$ & 0.0640 & 0.9 & 0.8720 & $0 \cdot 05637$ & $1 \cdot 0185$ & 401 \\
\hline $1 \frac{1}{8}$ & 9 & $0 \cdot 1111$ & $0 \cdot 0711$ & & 0.9828 & $0 \cdot 06263$ & $1 \cdot 1459$ & 0.9473 \\
\hline $1 \frac{1}{4}$ & 9 & $0 \cdot 1111$ & 0.0711 & $1 \cdot 17$ & $1 \cdot 1078$ & 0.06263 & $1 \cdot 2709$ & 1.0723 \\
\hline 13 & 8 & 0.1250 & 0.0800 & $1 \cdot 2950$ & $1 \cdot 2150$ & 0.07046 & $1 \cdot 3981$ & $1 \cdot 1750$ \\
\hline $1 \frac{1}{2}$ & 8 & $0 \cdot 1250$ & 0.0800 & $1 \cdot 4$ & $1 \cdot 3400$ & 7046 & $1 \cdot 5231$ & 000 \\
\hline $1 \frac{5}{8}$ & 8 & $0 \cdot 1250$ & 0.0800 & $1 \cdot 54$ & $1 \cdot 4650$ & 0.07046 & $1 \cdot 6481$ & 1.4250 \\
\hline $1 \frac{\mathrm{g}}{4}$ & 7 & $0 \cdot 1429$ & $0 \cdot 0915$ & $1 \cdot 6585$ & $1 \cdot 5670$ & 0.08053 & $1 \cdot 7763$ & 1.5214 \\
\hline 2 & 7 & $0 \cdot 1429$ & 0.0915 & 1.90 & $1 \cdot 8170$ & 0.08053 & $2 \cdot 0$ & $1 \cdot 7714$ \\
\hline $2 \frac{1}{4}$ & 6 & 0.1667 & $0 \cdot 1067$ & $2 \cdot 14$ & $2 \cdot 0366$ & 9395 & $2 \cdot 2807$ & $1 \cdot 9833$ \\
\hline $2 \frac{1}{2}$ & 6 & $0 \cdot 1667$ & $0 \cdot 1067$ & $2 \cdot 3933$ & $2 \cdot 2866$ & 0.09395 & $2 \cdot 5307$ & $2 \cdot 2333$ \\
\hline 23 & 6 & $0 \cdot 1667$ & $0 \cdot 1067$ & $2 \cdot 643$ & $2 \cdot 5366$ & 0.09395 & $2 \cdot 7807$ & $2 \cdot 4833$ \\
\hline 3 & 5 & $0 \cdot 2000$ & $0 \cdot 1281$ & $2 \cdot 87$ & $2 \cdot 7438$ & $0 \cdot 11274$ & $3 \cdot 0368$ & $2 \cdot 6800$ \\
\hline 31 & 5 & $0 \cdot 2000$ & 0.1281 & $3 \cdot 1219$ & $2 \cdot 9938$ & $0 \cdot 11274$ & $3 \cdot 2868$ & $2 \cdot 9300$ \\
\hline $3 \frac{1}{2}$ & $4 \frac{1}{2}$ & $0 \cdot 2222$ & $0 \cdot 1423$ & $3 \cdot 3577$ & $3 \cdot 2154$ & $0 \cdot 12526$ & $3 \cdot 5410$ & $3 \cdot 1445$ \\
\hline $3 \frac{3}{4}$ & $4 \frac{1}{2}$ & $0 \cdot 2222$ & $0 \cdot 1423$ & $3 \cdot 6077$ & $3 \cdot 4654$ & $0 \cdot 12526$ & $3 \cdot 7910$ & $3 \cdot 3945$ \\
\hline 4 & $4 \frac{1}{2}$ & 0.2222 & $0 \cdot 1423$ & $3 \cdot 8577$ & $3 \cdot 7154$ & $0 \cdot 12526$ & $4 \cdot 0410$ & $3 \cdot 6445$ \\
\hline $4 \frac{1}{2}$ & 4 & $0 \cdot 2500$ & $0 \cdot 1601$ & $4 \cdot 3399$ & $4 \cdot 1798$ & $0 \cdot 14092$ & $4 \cdot 5461$ & $4 \cdot 0999$ \\
\hline 5 & 4 & $0 \cdot 2500$ & $0 \cdot 1601$ & $4 \cdot 8399$ & & & $5 \cdot 0461$ & $4 \cdot 5999$ \\
\hline $5 \frac{1}{2}$ & $3 \frac{1}{2}$ & 0.2857 & $0 \cdot 1830$ & $5 \cdot 3170$ & $5 \cdot 1340$ & $0 \cdot 16105$ & $5 \cdot 5527$ & $5 \cdot 0428$ \\
\hline$f$ & $3 \frac{1}{2}$ & $0 \cdot 2857$ & 0.1830 & $5 \cdot 8170$ & $5 \cdot 6340$ & $0 \cdot 16105$ & $6 \cdot 0527$ & $5 \cdot 5428$ \\
\hline
\end{tabular}

